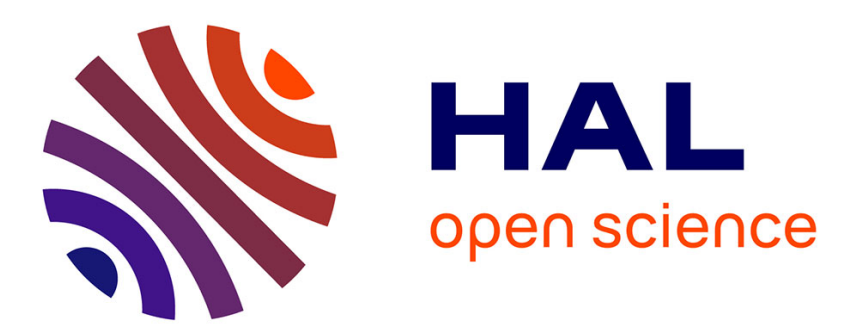

\title{
Thermal Stability of Medium- and High-Entropy Alloys of 3d-Transition Metals
}

A. Fourmont, A. S Rogachev, S. Le Gallet, O. Politano, D. Yu. Kovalev, N. A Kochetov, N. F Shkodich, S. G Vadchenko, Florence Baras

\section{- To cite this version:}

A. Fourmont, A. S Rogachev, S. Le Gallet, O. Politano, D. Yu. Kovalev, et al.. Thermal Stability of Medium- and High-Entropy Alloys of 3d-Transition Metals. Journal of Phase Equilibria and Diffusion, 2021, 42, pp.720. 10.1007/s11669-021-00903-y · hal-03347427

\section{HAL Id: hal-03347427 \\ https://hal.science/hal-03347427}

Submitted on 17 Sep 2021

HAL is a multi-disciplinary open access archive for the deposit and dissemination of scientific research documents, whether they are published or not. The documents may come from teaching and research institutions in France or abroad, or from public or private research centers.
L'archive ouverte pluridisciplinaire HAL, est destinée au dépôt et à la diffusion de documents scientifiques de niveau recherche, publiés ou non, émanant des établissements d'enseignement et de recherche français ou étrangers, des laboratoires publics ou privés. 
Journal of Phase Equilibria and Diffusion

Special issue: The Thermodynamics and Kinetics of High-Entropy Alloys

\title{
Thermal stability of medium- and high-entropy alloys of 3d-transition metals
}

\begin{abstract}
A. Fourmont ${ }^{1}$, A.S. Rogachev ${ }^{2}$, S. Le Gallet ${ }^{1}$, O.Politano ${ }^{1}$, D.Yu. Kovalev ${ }^{2}$, N.A. Kochetov ${ }^{2}$, N.F. Shkodich ${ }^{2}$, S.G. Vadchenko ${ }^{2}$ and F. Baras ${ }^{1, *}$

${ }^{1} I C B$ laboratory, UMR 6303 CNRS-Université Bourgogne Franche-Comté, 9 Av. Alain Savary, BP 47870, 21078 Dijon Cedex, France

${ }^{2}$ Merzhanov Institute of Structural Macrokinetics and Materials Science Russian Academy of Sciences, Chernogolovka Moscow region, Russia
\end{abstract}

\begin{abstract}
A family of High-Entropy Alloys (HEA) composed of 3d-transitional metals is attracting great interest due to the mechanical, magnetic and electrical properties that are valuable in the development of advanced structural and functional materials. In this work, we studied XCoCrFeNi HEAs, where $\mathrm{X}=\mathrm{Al}$, $\mathrm{Ti}$, or $\mathrm{Cu}$, produced by mechanical alloying by means of highenergy ball milling. Powder mixture composed of elemental metal powders transforms in a homogeneous alloy (super-saturated solid solution) due to the intense diffusion which takes place in the course of friction and shear deformation of the metal particles during the mechanical alloying. The thermal stability of the HEA phases was experimentally studied in the range $873 \mathrm{~K}$ - $1273 \mathrm{~K}$ using high-temperature X-ray diffraction and compared with the results of thermodynamic calculations (Calphad).
\end{abstract}




\section{Introduction}

Since their discovery by Yeh and Cantor ${ }^{1,2}$, HEAs have become a major research topic in material science. HEAs are characterized by outstanding properties including corrosion resistance, limited diffusion, increased plasticity at low temperatures, and other special mechanical and magnetic properties that depend on structure and composition. Nonetheless, their usage for technological purposes remains limited ${ }^{3}$. Prior to their future exploitation, a fundamental study of their stability is a required step. The stability of materials is of primary importance per se; indeed, reliable stability opens up new perspectives for applications including coatings, interconnects (fuel cell) ${ }^{4}$, and heat engine components ${ }^{5}$.

Typically, HEAs are characterized by a single-phase disordered solid solution. We may wonder if the absence of short- and long-range chemical order is maintained whatever the conditions. Is limited diffusion in a distorted crystal lattice the guarantee of this stability? What happens in the presence of defects or nanostructures that are inevitably formed during the elaboration process? One way to study the stability of a material is to submit it to annealing at high temperatures and look for possible structural transformations.

Thermal stability in cast and mechanically synthesized HEAs is debatable because experimental data are incomplete. Among the main high-entropy families, the family composed of 3d-transition metals is the most thoroughly studied. The CoCrFeNi single-phase alloy with an FCC crystal structure was characterized as stable at $1173 \mathrm{~K}$ for $600 \mathrm{~h}^{6}$, but the phase decomposition occurred at $1073 \mathrm{~K}$ for $800 \mathrm{~h}^{7}$. As-cast $\mathrm{Al}_{\mathrm{x}} \mathrm{CoCrFeNi}$ alloys commonly consist of FCC dendrites and a $\mathrm{BCC}$ interdendritic phase, depending on the Al content: only the FCC phase was obtained for $\mathrm{x}=0-0.3$, a $\mathrm{FCC}+\mathrm{BCC}$ mixture for $\mathrm{x}=0.5-0.8$, and $\mathrm{BCC}$ alone for $\mathrm{x}=0.9-3.0^{8,9}$. At temperatures above $873 \mathrm{~K}$, the as-cast equiatomic $\mathrm{AlCoCrFeNi} \mathrm{BCC}$ phase transforms into $\mathrm{FCC}$, with simultaneous precipitation of $\mathrm{B} 2$ and $\sigma$ phase inclusions ${ }^{10}$. A mechanically synthesized $\mathrm{AlCoCrFeNi}$ BCC 
phase transformed into a partially disordered B2 phase after $1 \mathrm{~h}$ annealing at $1073 \mathrm{~K}{ }^{11,12}$. Experimental data concerning phase transformations in $\mathrm{MnCoCrFeNi}, \mathrm{CuCoCrFeNi}$ and other HEAs have been reported in several reviews ${ }^{13-15}$. For instance, it has been shown that two similar FCC structures co-existed in an annealed milled $\mathrm{MnCoCrFeNi}{ }^{16}$; short-term annealing led to the release of $\mathrm{Cr}$ and $\mathrm{Mn}$ from the matrix, which shrunk and formed a second metastable FCC solid solution.

Equimolar TiCoCrFeNi is less often studied than the other compositions of the considered family. One would expect a very complex microstructure with several intermetallic phases due to Ti. However, TiCoCrFeNi after laser cladding was reported to exhibit one main FCC phase and two secondary Simple Cubic (SC) and Laves phases ${ }^{17}$.

In addition, the confrontation of experimental results with powerful modeling tools such as the Calphad method has proven useful. In their work, Zhang and Gao demonstrated that configurational entropy alone does not grant the stability of high-entropy alloys; thus, quantities such as phase enthalpy (whose calculation is made possible by Calphad) are of great interest in evaluating the stability of alloys in regard to chemical and temperature variations ${ }^{18,19}$. The chemical stability of the FCC solid solution in the MnCoCrFeNi system was thoroughly assessed and demonstrated to be very well described by a database such as TCHEA1, although an accurate secondary phase prediction was perfectible ${ }^{20}$. Thanks to this combined experimental and thermodynamic approach, conclusive results have been obtained by Rao et $a l .{ }^{21}$. They were able to efficiently identify nano-scale precipitates predicted by thermodynamic calculations and to confirm the role of $\mathrm{Al}$ in the phase formation in $\mathrm{Al}_{\mathrm{x}} \mathrm{CoCrFeNi}$ alloys.

In this work, we considered the family of HEAs composed of 3d-transition metals: CoFeNiXZ where $\mathrm{X}=\mathrm{Cr}$ and $\mathrm{Z}=\mathrm{Al}$, Ti. This family has been extensively studied since the discovery of the Cantor MnCoCrFeNi HEA. Starting from the system CoFeNi that forms the core of the alloy 
family, we considered the quaternary $\mathrm{CoCrFeNi}$ and finally quinary systems. We focused on HEAs produced by mechanical alloying. This production method has rapidly developed over the last decade 22,23 . Here, the alloying is carried out by means of high-energy planetary ball milling (HEBM) of powder mixtures composed of elemental metallic powders. The alloyed powders were characterized with High Temperature X-ray Diffraction (HT-XRD) and room temperature XRD after annealing in order to estimate their stability.

We also carried out the thermodynamic description of ternary, quaternary and quinary systems using the Calphad approach. In this way, we were able to interpret the experimental results as compared to expected equilibrium phases and to evaluate the specificities of the alloying process followed by annealing. 


\section{Materials and methods}

Elemental powders of $\mathrm{Al}, \mathrm{Ti}, \mathrm{Cu}, \mathrm{Co}, \mathrm{Cr}, \mathrm{Fe}$ and $\mathrm{Ni}$ of purity greater than $98 \mathrm{wt} \%$ and of various particle sizes (all $<160 \mu \mathrm{m})$ were mixed in equimolar proportions and mechanically alloyed in high-energy planetary ball mills. Table 1 summarizes the structure and the lattice parameter of the metallic element used in this work. A water-cooling mill “Activator-2S" (Novosibirsk, Russia) with stainless-steel cylindrical jars and balls $(7 \mathrm{~mm}$ in diameter) was used for most of the systems studied in this work. In these cases, the jars were evacuated and then filled with Ar gas at 4 bar. The HEBM was run at a rotating speed of the sun wheel and the grinding drums at 694 and 1388 rpm, respectively. TiCoCrFeNi was obtained using the "AGO-2" (Novosibirsk, Russia) watercooling planetary mill with steel balls $(9 \mathrm{~mm}$ in diameter) in air atmosphere, at a rotating speed of $912 \mathrm{rpm}$ (for the mill) that corresponded to $2220 \mathrm{rpm}$ for the jars. The second milling device was used in order to prove that presence of oxygen in a milling jar does not prevent formation of singlephase multicomponent alloys. The ball/powder weight ratio was 20:1 in all experiments. The milling time was systematically 90 minutes, or 120 minutes for the $\mathrm{CuCoCrFeNi}$ system.

The X-ray diffraction analysis of powders was performed with a DRON-3M diffractometer (Russia). Both $\mathrm{Cu}-\mathrm{K}_{\alpha}$ radiation (wavelength $\lambda=0.15419 \mathrm{~nm}$ ) and $\mathrm{Fe}-\mathrm{K}_{\alpha}$ radiation $(\lambda=0.19374$ $\mathrm{nm}$ ) were used in order to obtain complementary data. Indeed, the Fe- $\mathrm{K}_{\alpha}$ ensures a better angle resolution. High-temperature XRD measurements were made in a vacuum at $10^{-3} \mathrm{~Pa}$, within the temperature range 298-1273 K using an ARL'XTRA diffractometer with the high- temperature accessory HTK2000 “Anton Paar” (Switzerland). The selected annealing temperatures were 873 $\mathrm{K}, 1073 \mathrm{~K}$ and $1273 \mathrm{~K}$. For the accurate measurement of lattice parameters at room temperature (before and after annealing), crystalline silicon powder (NIST SRM 640b) admixed to the analyzing powder samples was used as inner marker (standard). The lattice parameters were determined with accuracy better than $0.002 \%$. The SEM and EDS analyses were carried out using a Zeiss Ultra Plus field-emission scanning electron microscope (Carl Zeiss, Germany), 
equipped with an INCA Energy 350 XT energy dispersive spectrometer (Oxford Instruments, UK).

Thermodynamic calculations were performed using Thermo-Calc ${ }^{\circledR}$ software based on the Calphad methodology with the TCHEA3 database. Phase fractions and phase composition as a function of temperature were calculated for the different systems. The lattice parameter of the cubic solid solution was estimated in the cases of ternary and quaternary systems.

\section{Results}

\subsection{CoFeNi and CoCrFeNi}

Three metals of the iron group, $\mathrm{Co}, \mathrm{Fe}$, and $\mathrm{Ni}$ constitute a ternary core of the $3 \mathrm{~d}$-transition metal family of HEAs. These metals have similar atomic radii, but different crystal structures.

Figures 1 and 2 display the dependence of the equilibrium phase composition (expressed in volume ratio) on temperature in the case of the ternary $\mathrm{CoFeNi}$ and quaternary $\mathrm{CoCrFeNi}$. In order to get a quick estimation of the composition of each phase, we also provide the distribution of elements in the different phases. The calculation was here performed in the case of equimolar systems. For the ternary $\mathrm{CoFeNi}$, the main characteristics are as follows:

- Below $925 \mathrm{~K}$, the system is composed of one ordered BCC phase and one disordered FCC phase. Nickel is the principal element in the FCC phase. The BCC ordered phase contains mainly Co and Fe elements.

- Above $925 \mathrm{~K}$, a single FCC disordered phase is observed.

- $\quad$ The melting temperature is $1734 \mathrm{~K}$.

In conclusion, the ternary CoFeNi may be considered as an HEA with only 3 elements. 
This typical behavior can be examined in light of the behavior of pure elements and binary phase diagrams. Nickel possesses an FCC structure from room temperature to its melting point. Although $\mathrm{Co}(\mathrm{Fe})$ has an $\mathrm{HCP}(\mathrm{BCC})$ structure at room temperature, $\mathrm{Co}(\mathrm{Fe})$ undergoes a phase transformation to FCC above $695 \mathrm{~K}(1184 \mathrm{~K})$. The binary phase diagrams show that a single FCC solid solution $\mathrm{Co}+\mathrm{Ni}$ or $\mathrm{Fe}+\mathrm{Ni}$ can be obtained in a wide range of temperatures for any ratio between the elements. On the other hand, Co and Fe form a BCC solid solution below $1173 \mathrm{~K}$ if the mole fraction in Fe is greater than 0.2 or a FCC solid solution at a lower content in Fe. It is therefore not surprising that a single disordered FCC solid solution is observed at high temperatures.

Adding the element $\mathrm{Cr}$ to obtain the quaternary system CoCrFeNi leads to the following observations:

- Above $928 \mathrm{~K}$, the system is composed of a single FCC disordered phase corresponding to the HEA.

- At lower temperatures [800 K-928 K], three phases coexist: a BCC-ordered phase with Co and $\mathrm{Fe}$, a $\sigma$ phase with a majority of $\mathrm{Cr}$ and the disordered FCC solid solution.

- $\quad$ The melting temperature is $1720 \mathrm{~K}$.

Ternary and quaternary systems are characterized by the same melting temperatures. In equilibrium conditions, a single FCC solid solution is formed in a temperature range close to the melting temperature. For lower temperatures, the equilibrium corresponds to a mixture of different phases.

Fig. 3 displays the XRD patterns of CoFeNi and CoCrFeNi after 90 min of high-energy ball milling (HEBM). At the end of the process, Co, Fe and $\mathrm{Cr}$ peaks have disappeared. There remain only two peaks corresponding to an FCC structure. The broadening of these two peaks is easily noticeable; this broadening signifies that the powder most likely contains a high concentration of lattice 
defects and nanosized crystallites. Indeed, a crystalline size (coherent-scattering regions) was roughly estimated as $15 \mathrm{~nm}$ for $\mathrm{CoFeNi}$ and $21 \mathrm{~nm}$ for $\mathrm{CoCrFeNi}$ after HEBM. In addition, there is a strong shift on the FCC peaks; for instance, for CoFeNi, the (200) FCC peak jumps from $66.7^{\circ}$ $2 \theta$ (corresponding to the pure Ni lattice parameter $\mathrm{a}_{0}=3.520 \AA$ ) to $65.6^{\circ} 2 \theta$. The lattice parameters of the FCC phase after $90 \mathrm{~min}$ of HEBM are estimated to be $3.57 \AA$ for CoFeNi and $3.58 \AA$ for $\mathrm{CoCrFeNi}$. Hence, there is a strong lattice parameter increase associated with the formation of an FCC solid solution. The calculated lattice parameters are summarized in Table 2.

SEM images of milled powder cross sections are shown in Fig. 4. The lack of contrast demonstrates that only one chemically uniform phase remains after milling. EDS microanalysis reveals that the global composition is still equimolar in $\mathrm{CoFeNi}$ and $\mathrm{CoCrFeNi}$. There is no preferential adherence to the milling apparatus by any one element in particular. From XRD measurements and SEM analyses, we can conclude that the mixtures of the individual powders $\mathrm{Co}+\mathrm{Fe}+\mathrm{Ni}$ and $\mathrm{Co}+\mathrm{Cr}+\mathrm{Fe}+\mathrm{Ni}$ have completely transformed into the single-phase alloys $\mathrm{CoFeNi}$ and $\mathrm{CoCrFeNi}$.

The CoFeNi and CoCrFeNi milled powders were annealed at $873 \mathrm{~K}, 1073 \mathrm{~K}$ and $1273 \mathrm{~K}$ over 5.1 h. The XRD patterns at room temperature after annealing are presented in Fig. 5.

Two minor peaks at $41.5^{\circ} 2 \theta$ and $50.25^{\circ} 2 \theta$ probably correspond to iron-chromium oxide $(\mathrm{Fe}, \mathrm{Cr})_{2} \mathrm{O}_{3}$ (card PDF 000-34-0412). Although the samples were heated in a vacuum during the high-temperature XRD analysis, some residual oxygen results in surface oxidation of the powder particles. There is no sign of any other phase formation during annealing, although we could have expected the formation of a $\sigma$ phase in the case of the CoCrFeNi alloy. The direct comparison of thermodynamic calculation to experimental results thus leads to the conclusion that the HEBM process selects the HT phase: the FCC disordered solid solution. However, there is a slight 
decrease in lattice parameter with temperature in the case of CoFeNi whereas there is a slight increase in the case of $\mathrm{CoCrFeNi}$ (see Table 2). Note that this does not correspond to thermal expansion since these XRD measurements are performed at room temperature. Rogachev et al. suggested that the decrease could be due to the relaxation of the crystal structure ${ }^{16}$. The increase in lattice parameter after annealing of the milled powder seems to be related to Cr. It may suggest that some Cr-rich regions remained after milling, and that annealing led to a second chemical homogenization associated with a lattice parameter increase. Indeed, $\mathrm{Cr}$ has the highest melting temperature among the 4 considered elements and is known to be the last element to dissolve during milling. It could also be due to the formation of the Fe,Cr oxides during annealing which would lower the actual concentrations of these elements in the alloy. A third hypothesis is linked to the fact that the (111) FCC peak shifts to the left whereas the (200) peak seems to remain stationary. This could be the sign of a weak, thermally induced martensitic transformation; indeed Rogachev et al. suggested that this kind of transformation could occur in the AlCoCrFeNi alloy under similar constraints ${ }^{24}$.

In addition, the width of the peaks narrows. This narrowing means that lattice defects are relaxing and that grain size is increasing. After annealing at $1273 \mathrm{~K}$, the sizes of coherent-scattering regions increase to $1340 \mathrm{~nm}$ and $680 \mathrm{~nm}$ for CoFeNi and CoCrFeNi, respectively.

The lattice parameter was estimated by XRD at annealing temperatures during the heat treatment for the ternary and quaternary (see Fig. 6). These experimental results have been compared to those obtained via two different theoretical approaches. The first estimation was directly calculated using Thermo-Calc. The second estimation relies on the well-known Vegard's law. Vegard's law is the empirical rule that the lattice parameter of a solid solution of two constituents is approximately equal to a rule of mixtures of the two constituent lattice parameters at the same temperature. If generalized to a ternary equimolar system:

$$
\mathrm{a}_{0}(\mathrm{CoFeNi})=\frac{1}{3}\left(\mathrm{a}_{0}(\mathrm{Co})+\mathrm{a}_{0}(\mathrm{Fe})+\mathrm{a}_{0}(\mathrm{Ni})\right)
$$


provided that all elements have the same crystallographic structure. Here, we used Thermo-Calc to obtain $\mathrm{a}_{0}(\mathrm{FCC}-\mathrm{Ni}, \mathrm{T}), \mathrm{a}_{0}(\mathrm{FCC}-\mathrm{Co}, \mathrm{T})$, and $\mathrm{a}_{0}(\mathrm{FCC}-\mathrm{Fe}, \mathrm{T})$ for pure elements. In the case of the ternary, we noted that the Vegard's value best reflects the experimental observations, while the thermodynamic approach overestimates the lattice parameter at high temperatures. In the case of the quaternary, both estimations give greater values than the observed lattice parameter. Overall, both calculation methods give results that are relatively close to experimental measurements; they have proven to be useful tools in evaluating lattice parameters at elevated temperatures.

\subsection{Quinary systems}

The volume ratios for $\mathrm{X}-\mathrm{CoCrFeNi} \mathrm{HEAs}$ were calculated using Thermo-Calc with the database TCHEA3 over a range of temperatures $[773 \mathrm{~K}-1773 \mathrm{~K}]$. Despite the fact that $\mathrm{Mn}-\mathrm{CoCrFeNi}$ is not considered in the present study, the phase fraction of the Cantor HEA is depicted in Fig. 7 for the sake of comparison. At low temperatures, there is coexistence between a $\sigma$ phase and a disordered FCC solid solution. At $773 \mathrm{~K}$, the Mn element is equally distributed in the $\sigma$ phase and FCC_dis. As the temperature increases, the volume fraction of the $\sigma$ phase decreases until complete disappearance at $1100 \mathrm{~K}$ as compared to $900 \mathrm{~K}$ in the quaternary $\mathrm{CoCrFeNi}$. The melting temperature, $1573 \mathrm{~K}$, is lower than the melting temperature of $\mathrm{CoCrFeNi}$.

As shown in Fig. 8a, Al-CoCrFeNi is characterized by the presence of a BCC_ord phase over the whole temperature range $[773 \mathrm{~K}-1773 \mathrm{~K}]$. At $1073 \mathrm{~K}$, the composition of the two coexisting phases reads:

- BCC_ord: $\mathrm{Al}_{28} \mathrm{Co}_{22} \mathrm{Fe}_{17} \mathrm{Ni}_{27}$ and

- $\quad \sigma: \mathrm{Co}_{15} \mathrm{Cr}_{55} \mathrm{Fe}_{26}$.

Above $1273 \mathrm{~K}$, the $\sigma$ phase disappears in favor of a BCC_dis phase. The chromium element is redistributed between the two $\mathrm{BCC}$ phases. At $1273 \mathrm{~K}$, we have

- BCC_ord: $\mathrm{Al}_{25} \mathrm{Co}_{21} \mathrm{Cr}_{11} \mathrm{Fe}_{18} \mathrm{Ni}_{25}$ 
- BCC_dis: $\mathrm{Co}_{17} \mathrm{Cr}_{45} \mathrm{Fe}_{26}$.

Note that the composition of the BCC_dis is similar to that of the $\sigma$ phase. In the equimolar case, only a very small fraction of FCC_dis is formed ( $0.1 \%$ in volume). A slight variation in the composition in Al leads to a very different result with the formation of an FCC_dis phase.

High Temperature XRD (HT-XRD) results on milled Al-CoCrFeNi powder are presented in Fig. 8b. Heating was carried out in a step-like manner with isothermal stages at 873,1073 , and $1273 \mathrm{~K}$. Starting from the bottom, the first pattern corresponds to room temperature XRD prior to annealing. The next three patterns correspond to XRD measurement at $873 \mathrm{~K}$ after $0 \mathrm{~h}, 1 \mathrm{~h}$ and $2 \mathrm{~h}$. The next 6 patterns follow the same logic; the final XRD pattern corresponds to room temperature measurement after annealing.

After HEBM and prior to annealing, the Al-CoCrFeNi powder alloy seems to exhibit a single BCC phase, similarly to what was reported by $\mathrm{Ji}$ et al. with a far less powerful milling ${ }^{25}$. However, a mixture of FCC+BCC has also been reported in several studies on the elaboration process of AlCoCrFeNi by powder metallurgy ${ }^{26,27}$. In addition to the influence of the mechanical alloying on the phase constitution of the alloys, initial elemental particle sizes could play a major role in determining the final crystalline structure after HEBM. It is worth noting that the only peak remaining after HEBM is very broad. This peak may actually correspond to two peaks: the (111) FCC and (110) BCC, both situated around $44.5^{\circ} 2 \theta$; however, the (200) FCC peak is completely absent from the XRD pattern.

The HT-XRD patterns show that the single BCC phase is stable up to $1273 \mathrm{~K}$. From this temperature, an FCC and an intermetallic $\sigma$ phase begin to form. Then, close analysis of the $2 \mathrm{~h}$ annealing pattern at $1273 \mathrm{~K}$ may suggest that there is a partial dissolution of $\sigma$ in the FCC phase. After cooling, three phases remain: FCC, BCC and $\sigma$. The agreement between experimental and calculation results is not excellent but leads to several remarks. Indeed, the calculated domain of stability of $\sigma$ seems to be slightly overestimated since there is no sign of $\sigma$ at $873 \mathrm{~K}$ and since $\sigma$ 
begins to disappear at $1273 \mathrm{~K}$ although its calculated domain was [817 K-1276 K]. Obviously, one could argue that $2 \mathrm{~h}$ is not enough to reach thermodynamic equilibrium and that the $\sigma$ phase prediction is known to be perfectible ${ }^{20}$. However, the formation of an FCC phase is barely expected according to the database and this constitutes a major discrepancy. The formation of an FCC phase could be partially explained by a composition modification due to oxide formation ${ }^{28}$. Indeed, oxide formation is a well-known drawback of the powder metallurgy route and could lead to an $\mathrm{Al}$ depletion in the main phase.

As shown in Fig. 9a, the volume ratios for $\mathrm{Cu}-\mathrm{CoCrFeNi} \mathrm{HEA}$ were calculated using ThermoCalc with the database TCHEA3 over a range of temperatures $[773 \mathrm{~K}-1773 \mathrm{~K}]$. This HEA is characterized by the coexistence of two disordered FCC solid solutions at high temperatures (T > $973 \mathrm{~K})$ :

- FCC_dis\#1 with a majority of $\mathrm{Cu}\left(\mathrm{Cu}_{89} \mathrm{Co}_{1} \mathrm{Fe}_{2} \mathrm{Ni}_{8}\right)$

- FCC_dis\#2 without $\mathrm{Cu}\left(\mathrm{Cu}_{2} \mathrm{Co}_{25} \mathrm{Cr}_{25} \mathrm{Fe}_{25} \mathrm{Ni}_{23}\right)$.

The FCC_dis\#1 phase melts prior to the appearance of the? FCC_dis\#2 phase. The amount of FCC_dis\#1 is directly related to the fraction of $\mathrm{Cu}$. The ordered $\mathrm{BCC} \_$ord phase and $\sigma$ phase are observed at relatively low temperatures.

HT-XRD results on milled $\mathrm{Cu}-\mathrm{CoCrFeNi}$ powder are presented in Fig. 9b. Measurements were carried out slightly differently than in the Al-CoCrFeNi case, the sample were heated at the selected annealing temperature over $5 \mathrm{~h}$ and then cooled down for each temperature of interest. For the sake of comparison, the first pattern corresponds to XRD results after $1 \mathrm{~h}$ of annealing and the second after $2 \mathrm{~h}$. Mechanical treatment of the five-component $\mathrm{Cu}+\mathrm{Co}+\mathrm{Cr}+\mathrm{Fe}+\mathrm{Ni}$ mixtures results in the formation of a single-phase FCC structure as shown in the bottom pattern.

The HT-XRD patterns reveal that a small amount of a BCC phase appears at $873 \mathrm{~K}$ but disappears at $1073 \mathrm{~K}$ and above. This BCC phase is predicted by Calphad but at a lower temperature and could be the sign that its stability domain is shrunk by the consequences of the elaboration process 
(such as contamination or high concentration of lattice defects). Indeed, a high concentration of lattice defects tends to promote new phase formation. A second FCC phase, noted FCC-1, appears at $1073 \mathrm{~K}$ after $2 \mathrm{~h}$ of annealing, remains at $1273 \mathrm{~K}$ after $1 \mathrm{~h}$ but disappears for longer annealing times and is not visible after cooling. However, the two FCC phases could have very similar lattice parameters and appear as one XRD pattern. According to our thermodynamic calculations, a minor $\mathrm{Cu}$-rich FCC is supposed to exist below $1393 \mathrm{~K}$. The lattice parameter of the $\mathrm{Cu}$-rich $\mathrm{FCC}$ phase is estimated at $3.70 \AA$ at $1273 \mathrm{~K}$ by Thermo-Calc, whereas the lattice parameter of the main FCC phase is estimated at $3.64 \AA$. Hence, the hypothesis of two lattices of the same parameter seems unlikely.

In the case of the Ti-CoCrFeNi, Calphad showed the coexistence of a disordered FCC_dis solid solution and a BCC_ord phase close to the melting temperature (see Fig. 10a). The $\sigma$ phase persists upon melting. At $1273 \mathrm{~K}$, the composition of the three coexisting phases reads:

- BCC_ord: $\mathrm{Ti}_{28} \mathrm{Co}_{21} \mathrm{Cr}_{14} \mathrm{Fe}_{16} \mathrm{Ni}_{21.5}$;

- FCC_dis: $\mathrm{Ti}_{5} \mathrm{CO}_{20} \mathrm{Cr}_{28} \mathrm{Fe}_{28} \mathrm{Ni}_{19}$;

- $\quad \sigma: \mathrm{Co}_{15} \mathrm{Cr}_{53} \mathrm{Fe}_{24}$.

Most of the Ti is in the BCC_ord phase. At low temperatures $(\mathrm{T}<1000 \mathrm{~K})$, Laves phases C14 and $\mathrm{C} 15$, intermetallic $\mathrm{Ni}_{3} \mathrm{Ti}$ (D024) and the $\sigma$ phase are stable. Laves phases and intermetallic $\mathrm{Ni}_{3} \mathrm{Ti}$ rearrange at $1000 \mathrm{~K}$ into $\mathrm{FCC}$ _dis and $\mathrm{BCC}$ _ord. The melting of FCC_dis takes place prior to the melting of the BCC_ord. Over $40 \mathrm{~K}$, the ordered BCC_ord phase coexists with the liquid phase.

HT-XRD results on milled Ti-CoCrFeNi powder are presented in Fig. 10b. Heating was carried out similarly to the previous $\mathrm{Al}-\mathrm{CoCrFeNi}$ alloy, except that the XRD patterns after $0 \mathrm{~h}$ of annealing at the selected temperature are not displayed. Mechanical treatment of the fivecomponent $\mathrm{Ti}+\mathrm{Co}+\mathrm{Cr}+\mathrm{Fe}+\mathrm{Ni}$ mixtures results in the formation of a single-phase $\mathrm{BCC}$ structure 
as shown in the bottom pattern. In this case, the last remaining peak is also very broad. It could correspond to two peaks, one FCC and one BCC.

The HT-XRD patterns show that the peaks corresponding to the BCC and FCC phases at $44.5^{\circ} 2 \theta$ appear clearly as two separate peaks from $873 \mathrm{~K}$ and above. At $873 \mathrm{~K}$, the BCC phase is predominant, whereas the FCC phase becomes the main phase at 1073 and $1273 \mathrm{~K}$. Note that the $\mathrm{W}$ peak corresponds to the measurement apparatus. In this case, there is also a noticeable peak refinement with temperature, corresponding to the increase in grain size and relaxation of the lattice defects.

The superlattice peak associated with an ordered BCC phase B2 should be close to $32^{\circ} 2 \theta$. However, the main BCC peak, (110), is already so small that the superlattice reflection would not be visible either way. The inversion between BCC and FCC phases as the main phase of the Ti$\mathrm{CoCrFeNi}$ alloy as compared to calculated results is interesting; this discrepancy could be explained in a similar way to that noted in the Al-CoCrFeNi case. Indeed, Ti is known to be even more reactive with oxygen than $\mathrm{Al}$ and could form a significant amount of nano-oxide not discernible by XRD. However, our experimental results are coherent with laser cladded TiCoCrFeNi which exhibited a main FCC phase ${ }^{17}$. These results thus seem to point out a weakness in the TCHEA3 database.

\section{Discussion}

The stability of HEAs is typically associated to the relation

$$
\Delta G_{\mathrm{mix}}=\Delta H_{\mathrm{mix}}-T \Delta S_{\mathrm{mix}}
$$

It is commonly accepted that the entropic term ensures the stability of the alloy at high temperatures because of the increase in configurational entropy for a large enough number of constitutive elements. This simple and elegant point of view is currently called into question, 
justifying the name change of HEAs to complex concentrated alloys (CCAs) ${ }^{18,29}$. In order to appreciate the contribution of enthalpy and entropy in HEA stability (eq. (2)), the mixing behavior in Gibbs free energy, enthalpy, and entropy is presented in Fig. 11. We compared the mixing properties at high temperature $(1273 \mathrm{~K})$ of the quaternary $\mathrm{CoCrFeNi}$ and the quinaries $\mathrm{Al}-\mathrm{CoCrFeNi}$, and $\mathrm{Ti}-\mathrm{CoCrFeNi}$ for a given phase. In this analysis, the content in $\mathrm{Cr}$ has been varied. The diagrams depicted in Fig. 11 are pseudo-binary: there is an equal repartition of the other elements whatever the content in Cr. In our previous analysis, we have remarked that the distribution of $\mathrm{Cr}$ in the different phases is an important factor in the occurrence of disordered phases. In the case of the $\mathrm{CoCr}_{\mathrm{x}} \mathrm{FeNi}$ system, we noted that the stability $\Delta G_{\text {mix }}<0$ is ensured by eq. (2) because $\Delta H_{\text {mix }}>0$ in almost the whole composition range.

But the Gibbs free energy of mixing $\Delta G_{\text {mix }}$ decreases monotically over the whole composition range with the increase in the number of components (4 to 5). As shown in Fig. 11b(11e), the addition of $\mathrm{Al}(\mathrm{Ti})$ radically changes the behavior of $\Delta H_{\mathrm{mix}}$. Achieving a more negative $\Delta H_{\mathrm{mix}}$ implies the formation of stronger bonds among elements. This effect is very important at a low content in $\mathrm{Cr}$ (including the equimolar $x(\mathrm{Cr})=0.2$ ). In the case of the equimolar Al-CoCrFeNi system, $\Delta H_{\text {mix }}$ is about $-30 \mathrm{~kJ} / \mathrm{mol}$ for the BCC phase and $-15 \mathrm{~kJ} / \mathrm{mol}$ for the $\mathrm{FCC}$ phase. More surprisingly, we noted a decrease in $\Delta S_{\text {mix }}$ as compared to the quaternary system and the entropic term $T \Delta S_{\text {mix }}=4.67 \mathrm{~kJ} / \mathrm{mol}$. The stability of the quinary HEA thus cannot be solely attributed to the entropic effect. The same conclusions can be drawn in the case of the Ti-CoCrFeNi system.

The ternary CoFeNi system, often named medium-entropy alloy, in fact satisfies the relation (2) defining the notion of high-entropy alloy despite the limited number of constituents: $\Delta H_{\text {mix }}=$ $32.5 \mathrm{~kJ} / \mathrm{mol}>0 ;-T \Delta S_{\text {mix }}=-109 \mathrm{~kJ} / \mathrm{mol}$ at $T=1273 \mathrm{~K}$.

In the present work, the ternary elaborated by HEBM is composed of a single FCC disordered phase whose lattice parameter $a_{0}=3.57 \AA$ is greater than the lattice parameter of Ni $\left(a_{0}=3.52\right.$ 
$\AA$ ). Although the three constituents have similar atomic diameters, the host FCC-Ni matrix is affected by the substitution of $\mathrm{Ni}$ by $\mathrm{Co}$ and $\mathrm{Fe}$. This solid solution obtained by mechanical treatment is chemically uniform. The annealing of CoFeNi at $873 \mathrm{~K}, 1073 \mathrm{~K}$, and $1273 \mathrm{~K}$ has no effect on the phase stability except a slight relaxation of the lattice parameter measured at room temperature ( $a_{0}=3.56 \AA$ after the annealing at $1273 \mathrm{~K}$ for instance). In other words, the elaboration process selects the FCC_dis phase that remains stable upon annealing. Over the duration of annealing at $873 \mathrm{~K}$, no transformation of FCC_dis to BCC_ord was observed, while this ordered phase, mainly composed of $\mathrm{Fe}$ and $\mathrm{Co}$, is predicted in equilibrium conditions. Our hypothesis is that such discrepancies could be explained by the out of equilibrium process associated with powder metallurgy. The situation is completely different in the case of CoFeNi films prepared by reverse pulse electrodeposition with ultra-high magnetic saturation ${ }^{30}$. In this case, the $\mathrm{Co}_{20} \mathrm{Fe}_{66} \mathrm{Ni}_{14}$ alloy is unstable for $2 \mathrm{~h}$ annealing at $673 \mathrm{~K}$ with a significant reduction in magnetic saturation.

The quaternary CoCrFeNi system also fulfils relation (1). Fig. 11 demonstrates the High Entropy effect on alloy stability. The thermal stability of $\mathrm{CoCrFeNi}$ has recently attracted serious scrutiny in the literature. Indeed, this system can be considered as the base alloy for the class of $\mathrm{X}-\mathrm{CoCrFeNi} \mathrm{HEAs}$ where $\mathrm{X}$ is an element of different atomic size (Al or Ti in the present study). In a temperature range close to its melting temperature, at equilibrium, the system is characterized by a single FCC disordered phase (see Fig. 5). The thermal stability of the FCC_dis phase was investigated in systems produced by means of different techniques. In the case of HEAs prepared by arc-melting, Vaidya et al. did not observe any modification in structure or composition for a heat treatment in the temperature interval [1073 K-1373 K] for 96 $\mathrm{h}{ }^{31}$. On the other hand, Feng He et al. reported that the CoCrFeNi HEA is thermally metastable at $1023 \mathrm{~K}$ for a long annealing of $800 \mathrm{~h}^{32}$. The phase decomposition is similar to the clustering of GP zones with the formation of an FCC phase with a different lattice parameter $\left(a_{0}=3.55 \AA\right.$ 
as compared to the matrix value $a_{0}=3.66 \AA$ ). The thermal stability is thus revealed to be very sensitive to the duration and temperature of annealing. The existence of two very similar FCC_dis phases with a slight difference in lattice parameter $(0.001 \AA)$ has also been observed in non-equimolar as-cast $\mathrm{CoCrFeNi}$ with no change after 3 h-annealing at $1373 \mathrm{~K}^{33}$.

In the case of HEAs synthesized by mechanical alloying followed by spark plasma sintering (SPS), the situation is quite different. S. Praveen et al. reported that elemental powders of Co, $\mathrm{Cr}, \mathrm{Fe}$, and Ni milled for $15 \mathrm{~h}$ transformed into a major FCC_dis phase with a minor BCC_ord phase $^{6}$. After SPS, the major FCC phase was retained and the BCC_ord phase disappeared with the formation of tiny second phase particles (carbide $\mathrm{Cr}_{7} \mathrm{C}_{3}$ and oxide $\mathrm{Cr}_{2} \mathrm{O}_{3}$ ). Even for a long annealing duration of $600 \mathrm{~h}$ at $1173 \mathrm{~K}$, these authors observed sluggish grain growth that did not affect the stability of the nanocrystalline HEA alloy. The same behavior has been observed for long annealing at the lower temperature of $973 \mathrm{~K}^{34}$. The authors attributed the strong resistance to grain growth to the presence of carbides and oxides. In the present study, no such secondary phases were observed. Grain growth, estimated by the increase in crystallite size, is thus not inhibited by such oxides and carbides.

Nanocrystalline CoCrFeNi thin films deposited on $\mathrm{Si} / \mathrm{SiO} 2$ and c-sapphire substrates by magnetron co-sputtering have recently been investigated ${ }^{35}$. In addition to the FCC phase, the films contained an additional $\sigma$ phase even at room temperature and in as-deposited films due to a large number of nucleation sites and accelerated kinetics as compared to bulk systems. Annealing at temperatures between $573 \mathrm{~K}$ and $1073 \mathrm{~K}$ leads to the formation of $\mathrm{Cr}$ oxides at the expense of the $\sigma$ phase.

The powder of CoCrFeNi-HEA produced by HEBM (see Fig. 1) is characterized by a single FCC_dis solid solution of uniform chemical composition (see Fig. 2). The annealing at $873 \mathrm{~K}$, 
$1073 \mathrm{~K}$ and $1273 \mathrm{~K}$ over $5 \mathrm{~h}$ did not affect the stability of the disordered FCC phase, even at 873 $\mathrm{K}$ where thermodynamics predicts the formation of a small amount of $\sigma$ phase. The only noticeable change was an increase in the lattice parameter that may be related to the complete dissolution of $\mathrm{Cr}$ in the solid solution upon heat treatment or to the other hypotheses raised in section 3. In conclusion, two main factors may induce discrepancies between experimental and numerical results: the metastable state attained after powder metallurgy route and contamination by minor elements. Both HEBM preparation and annealing conditions are of primary importance in the observed phase stability.

The equimolar Al-CoCrFeNi system is among the most studied HEAs after the Cantor alloy. Nevertheless, discrepancies between experimental results and thermodynamic calculations have continuously been reported. In our study, a suspicious amount of FCC phase was found as soon as the temperature reached $873 \mathrm{~K}$. The hypothesis of $\mathrm{Al}$ oxide depleting the alloys of $\mathrm{Al}$ may partially explain this observation, but it is not sufficient. Indeed, we can turn to Munitz et al. who focused their work on the effect of heat treatment on the arc-melted Al-CoCrFeNi alloy ${ }^{36}$. The as-cast alloy exhibited a BCC phase $(\mathrm{A} 2+\mathrm{B} 2)$ with an $\mathrm{Al}-\mathrm{Ni}$ rich dendrite core and $\mathrm{Co}-\mathrm{Cr}-\mathrm{Fe}$ rich interdentritic regions. A $\sigma$ phase and an FCC phase formed in the interdendritic regions with heat treatments between $923 \mathrm{~K}$ and $1248 \mathrm{~K}$ over $3 \mathrm{~h}$. The formation of $\mathrm{Al}$ oxide is easier to control in the case of the liquid-state process; the influence of oxygen is thus most likely negligible in this study. Yet the amount of FCC after annealing is significant; the database seems unable to predict the correct equilibrium in this case. It is interesting to note that TCNI9, a database very similar to HEA3, predicts a greater amount of FCC.

Studies of $\mathrm{Cu}-\mathrm{CoCrFeNi}$ HEAs are mainly limited to thin films and coatings. An et al. have found that thin films were composed of FCC grains with preferential (111) orientation as compared to as-cast $\mathrm{Cu}$-CoCrFeNi HEAs where two FCC phases were detected (3.58 $\AA$ and $3.61 \AA$ ): a Cu-rich 
phase in interdendritic regions and a $\mathrm{Cu}$-poor phase in dendritic morphology ${ }^{37}$. The in-situ annealing in TEM of $\mathrm{Cu}-\mathrm{CoCrFeNi}$ thin films prepared by magnetron sputtering has been evaluated in ${ }^{38}$ in the temperature range [ $673 \mathrm{~K}-973 \mathrm{~K}$ ] for $5 \mathrm{~min}$. Up to $673 \mathrm{~K}$, a single FCC phase was observed. At $823 \mathrm{~K}$, the film had a two-phase structure: the original FCC phase and a BCC phase with an epitaxial relationship. This type of transformation can be attributed to diffusionless processes. From $823 \mathrm{~K}$, fast morphological and structural changes occur, controlled by volume diffusion processes with the separation of $\mathrm{Cr}$-rich areas and $\mathrm{Cu}$-rich ones. In the present study, the annealing of $\mathrm{Cu}-\mathrm{CoCrFeNi} \mathrm{HEA}$ produced by HEBM also revealed transient phases: a BCC phase and a second FCC phase as a function of temperature and annealing time.

Equimolar Ti-CoCrFeNi is among the least studied HEAs. Indeed, several groups evaluated the influence of $\mathrm{Ti}$ on the $\mathrm{CoCrFeNi}$ base ${ }^{39-41}$ and they concluded that $\mathrm{Ti}$ often leads to complex phases such as laves and rhombohedral phases. Surprisingly enough, in our study we found only two simple phases: FCC and BCC. The main difference is related to grain size and lattice defect concentration. Indeed, milled powders are known to exhibit a loose crystalline structure and complex phases could be kinetically inhibited. Hence, long term annealing could be mandatory to reveal the actual microstructure of this alloy.

There is no consensus in the literature on the relevant duration of annealing. Indeed, annealing studies are performed over various durations. Long term annealing has been reported by several groups for a minimal annealing time of $50 \mathrm{~h}$ or even $100 \mathrm{~h}$ to ensure that the thermodynamic equilibrium is reached in HEAs ${ }^{19,42}$. Reynolds et al. performed high-energy synchrotron radiation X-ray diffraction measurements during in situ annealing (SR-XRD) and noted that $3 \mathrm{~h}$ annealing was sometimes not long enough ${ }^{43}$. As compared to the above-mentioned studies, the annealing times of a few hours as used in the present work may seem a little short. However, conclusive remarks have been drawn in the literature reporting studies with short annealing times. Indeed, Dolique et al. demonstrated that HEA thin films deposited by DC magnetron 
sputtering were stable until a temperature just below the bulk HEA degradation temperature using HT-XRD with short time annealing ${ }^{44}$. Even in their SR-XRD study, Reynolds et al. concluded that their experimental results matched well with the Calphad predictions of the phase evolution for the B2 phase. Hence, short annealing times are an efficient way to ensure that the database and experimental results match well enough to proceed to a thorough analysis characterized by hundreds of hours annealing time.

The Calphad method, based on free energy minimization, gives the dependence of the equilibrium phase composition on temperature. For the compositions investigated in the present study, the disordered phase characteristic of high entropy alloys is formed in a temperature range close to the melting point, while ordered phases correspond to lower temperatures. We observed that some of these secondary phases transitorily appeared during heat treatment while others were inhibited. In order to report these observations, the kinetics of phase transformations should be taken into account with a detailed description of precipitation and diffusion mechanisms together with thermodynamic parameters (driving forces) ${ }^{45}$.

\section{Conclusion}

The investigation of thermal stability in High Entropy Alloys is a vast and complex domain. On one hand, the name of these alloys has been shown to be somewhat misleading since the high entropy effect has been demystified by several research groups. On the other hand, the field of High Entropy Alloys includes a wide range of compositions and elaboration processes. In our study, we chose to start from ternary medium entropy alloys to progress to the well-known Al$\mathrm{CoCrFeNi}$ and the less studied $\mathrm{Ti}-\mathrm{CoCrFeNi}$. The three studied quinary compositions are relatively simple since they are equimolar with only one element varying. The thermal stability of mechanically alloyed powders is of great interest since they embody the perfect disordered solid solution; indeed, they do not exhibit contamination such as carbon from the sintering medium or segregation occurring during solidification. 
In the present study, we found that medium entropy alloys CoFeNi and $\mathrm{CoCrFeNi}$ were the most stable of the alloys studied. In addition, our results revealed that adding a fifth element irremediably led to the formation of secondary phases after and/or during annealing. In a further finding of relevance, even the most stable HEA, the Mn-CoCrFeNi Cantor alloy, has recently been reported to exhibit two FCC solid solutions after short time annealing ${ }^{16}$.

The limited diffusion in a distorted crystal lattice does not seem to guarantee the thermal stability of High Entropy Alloys. Indeed, the single-phase microstructure is lost after even a short annealing time. In addition, those secondary phases were not often predicted by the Calphad method based on TCHEA3 (Thermo-Calc). It seems likely that the presence of defects related to the elaboration process led to some sort of kinetic shifting of their stability domain. However, in the case of medium entropy alloys, by confronting the thermodynamic calculations and the HTXRD results, we observed that some phases were inhibited at relatively low temperatures. And, interestingly enough, in the case of quinary high entropy alloys, we found that complex phases such as Laves and other ordered phases could also be inhibited.

Experimental results demonstrated that phase transformations and/or precipitations of secondary phases took place in all considered equimolar HEAs. Thermodynamic calculations also showed the co-existence of two or more phases at room and moderate temperatures, which is in agreement with eq. (2) since the entropy impact decreases with decreasing temperature. At the same time, we never observed complete decay of the initial HEA phase into simple ordered compounds (intermetallics, ternary ordered alloys, etc.). High entropy FCC and BCC phases remain after annealing in all studied materials, despite the transformations. Our findings allow us to conclude that the search for stable high-temperature alloys can be continued. 


\section{Acknowledgements}

The experimental portion of this work was undertaken with the financial support of the Russian Science Foundation (project no. 20-13-00277). Thermo-Calc calculations were supported by the Pilot-Hy project funded by the Regional Council of Bourgogne Franche-Comté, the FEDER and the EIPHI Graduate School (Contract ANR-17-EURE-0002). Pr. G. Caboche is also gratefully acknowledged for helpful discussions. 
(1) Yeh, J.-W.; Chen, S.-K.; Lin, S.-J.; Gan, J.-Y.; Chin, T.-S.; Shun, T.-T.; Tsau, C.-H.; Chang, S.-Y. Nanostructured High-Entropy Alloys with Multiple Principal Elements: Novel Alloy Design Concepts and Outcomes. Adv. Eng. Mater. 2004, No. $5,5$.

(2) Cantor, B.; Chang, I. T. H.; Knight, P.; Vincent, A. J. B. Microstructural Development in Equiatomic Multicomponent Alloys. Mater. Sci. Eng. A 2004, 375-377, p 213-218.

(3) Lu, Y.; Gao, X.; Jiang, L.; Chen, Z.; Wang, T.; Jie, J.; Kang, H.; Zhang, Y.; Guo, S.; Ruan, H.; Zhao, Y.; Cao, Z.; Li, T. Directly Cast Bulk Eutectic and Near-Eutectic High Entropy Alloys with Balanced Strength and Ductility in a Wide Temperature Range. Acta Mater. 2017, 124, p 143-150.

(4) Yeh, A.-C.; Chen, Y.-M.; Liu, C.-K.; Shong, W.-J. Development of an Advanced Bond Coat for Solid Oxide Fuel Cell Interconnector Applications. J. Power Sources 2015, 296, p 426-432.

(5) Chen, J.; Zhou, X.; Wang, W.; Liu, B.; Lv, Y.; Yang, W.; Xu, D.; Liu, Y. A Review on Fundamental of High Entropy Alloys with Promising High-Temperature Properties. $J$. Alloys Compd. 2018, 760, p 15-30.

(6) Praveen, S.; Basu, J.; Kashyap, S.; Kottada, R. S. Exceptional Resistance to Grain Growth in Nanocrystalline CoCrFeNi High Entropy Alloy at High Homologous Temperatures. $J$. Alloys Compd. 2016, 662, p 361-367.

(7) He, F.; Wang, Z.; Wu, Q.; Li, J.; Wang, J.; Liu, C. T. Phase Separation of Metastable CoCrFeNi High Entropy Alloy at Intermediate Temperatures. Scr. Mater. 2017, 126, p $15-19$.

(8) Wang, W.-R.; Wang, W.-L.; Yeh, J.-W. Phases, Microstructure and Mechanical Properties of AlxCoCrFeNi High-Entropy Alloys at Elevated Temperatures. J. Alloys Compd. 2014, 589, p 143-152.

(9) Butler, T. M.; Weaver, M. L. Oxidation Behavior of Arc Melted AlCoCrFeNi MultiComponent High-Entropy Alloys. J. Alloys Compd. 2016, 674, p 229-244.

(10) Meshi, L.; Linden, Y.; Munitz, A.; Salhov, S.; Pinkas, M. Retardation of the $\sigma$ Phase Formation in the AlCoCrFeNi Multi-Component Alloy. Mater. Charact. 2019, 148, p 171-177.

(11) Shivam, V.; Basu, J.; Pandey, V. K.; Shadangi, Y.; Mukhopadhyay, N. K. Alloying Behaviour, Thermal Stability and Phase Evolution in Quinary AlCoCrFeNi High Entropy Alloy. Adv. Powder Technol. 2018, 29 (9), p 2221-2230.

(12) Shivam, V.; Shadangi, Y.; Basu, J.; Mukhopadhyay, N. K. Evolution of Phases, Hardness and Magnetic Properties of AlCoCrFeNi High Entropy Alloy Processed by Mechanical Alloying. J. Alloys Compd. 2020, 832, p 154826.

(13) Miracle, D. B.; Senkov, O. N. A Critical Review of High Entropy Alloys and Related Concepts. Acta Mater. 2017, 122, 448-511. https://doi.org/10.1016/j.actamat.2016.08.081.

(14) Yeh, J.-W. Physical Metallurgy of High-Entropy Alloys. JOM 2015, 67 (10), p 2254-2261.

(15) Rogachev, A. S. Structure, Stability, and Properties of High-Entropy Alloys. Phys. Met. Metallogr. 2020, 121 (8), p 733-764.

(16) Rogachev, A. S.; Vadchenko, S. G.; Kochetov, N. A.; Rouvimov, S.; Kovalev, D. Y.; Shchukin, A. S.; Moskovskikh, D. O.; Nepapushev, A. A.; Mukasyan, A. S. Structure and Properties of Equiatomic CoCrFeNiMn Alloy Fabricated by High-Energy Ball Milling and Spark Plasma Sintering. J. Alloys Compd. 2019, 805, p 1237-1245.

(17) Zhang, S.; Han, B.; Li, M.; Zhang, Q.; Hu, C.; Niu, S.; Li, Z.; Wang, Y. Investigation on Solid Particles Erosion Resistance of Laser Cladded CoCrFeNiTi High Entropy Alloy Coating. Intermetallics 2021, 131, p 107111.

(18) Zhang, C.; Gao, M. C. CALPHAD Modeling of High-Entropy Alloys. In High-Entropy Alloys; Gao, M. C., Yeh, J.-W., Liaw, P. K., Zhang, Y., Eds.; Springer International Publishing: Cham, 2016; p 399-444. 
(19) Zhang, C.; Zhang, F.; Diao, H.; Gao, M. C.; Tang, Z.; Poplawsky, J. D.; Liaw, P. K. Understanding Phase Stability of Al-Co-Cr-Fe-Ni High Entropy Alloys. Mater. Des. 2016, 109, p 425-433.

(20) Bracq, G.; Laurent-Brocq, M.; Perrière, L.; Pirès, R.; Joubert, J.-M.; Guillot, I. The Fcc Solid Solution Stability in the Co-Cr-Fe-Mn-Ni Multi-Component System. Acta Mater. 2017, 128, p 327-336.

(21) Rao, J. C.; Diao, H. Y.; Ocelík, V.; Vainchtein, D.; Zhang, C.; Kuo, C.; Tang, Z.; Guo, W.; Poplawsky, J. D.; Zhou, Y.; Liaw, P. K.; De Hosson, J. Th. M. Secondary Phases in AlxCoCrFeNi High-Entropy Alloys: An in-Situ TEM Heating Study and Thermodynamic Appraisal. Acta Mater. 2017, 131, p 206-220.

(22) Torralba, J. M.; Alvaredo, P.; García-Junceda, A. High-Entropy Alloys Fabricated via Powder Metallurgy. A Critical Review. Powder Metall. 2019, 62 (2), p 84-114.

(23) Vaidya, M.; Muralikrishna, G. M.; Murty, B. S. High-Entropy Alloys by Mechanical Alloying: A Review. J. Mater. Res. 2019, 34 (5), p 664-686.

(24) Rogachev, A. S.; Kovalev, D. Yu.; Kochetov, N. A.; Shchukin, A. S.; Vadchenko, S. G. Evolution of Crystal Structure in High-Entropy AlCoCrFeNi Alloy: An in Situ HighTemperature X-Ray Diffraction Study. J. Alloys Compd. 2021, 861, p 158562.

(25) Ji, W.; Fu, Z.; Wang, W.; Wang, H.; Zhang, J.; Wang, Y.; Zhang, F. Mechanical Alloying Synthesis and Spark Plasma Sintering Consolidation of CoCrFeNiAl High-Entropy Alloy. J. Alloys Compd. 2014, 589, p 61-66.

(26) Rogachev, A. S.; Kochetov, N. A.; Panteleeva, A. V.; Kuskov, K. V.; Kovalev, D. Y.; Shchukin, A. S.; Vadchenko, S. G.; Scheck, Y. B. High-Energy Ball Milling and Spark Plasma Sintering of the CoCrFeNiAl High-Entropy Alloy. Metals 2020, 10 (11), p 1489.

(27) Fourmont, A.; Le Gallet, S.; Politano, O.; Desgranges, C.; Baras, F. Effects of Planetary Ball Milling on AlCoCrFeNi High Entropy Alloys Prepared by Spark Plasma Sintering: Experiments and Molecular Dynamics Study. J. Alloys Compd. 2020, 820, p 153448.

(28) Fourmont, A.; Le Gallet, S.; Hoummada, K.; Descoins, M.; Desgranges, C.; Politano, O.; Baras, F. Dual-Phase AlCoCrFeNi HEA: Reactive Spark Plasma Sintering, Atom Probe Tomography Investigation and Oxygen Contamination. To be published. 2021.

(29) Gorsse, S.; Couzinié, J.-P.; Miracle, D. B. From High-Entropy Alloys to Complex Concentrated Alloys. Comptes Rendus Phys. 2018, 19 (8), p 721-736.

(30) Tabakovic, I.; Venkatasamy, V. Preparation of Metastable CoFeNi Alloys with Ultra-High Magnetic Saturation $(\mathrm{Bs}=2.4-2.59 \mathrm{~T})$ by Reverse Pulse Electrodeposition. J. Magn. Magn. Mater. 2018, 9.

(31) Vaidya, M.; Guruvidyathri, K.; Murty, B. S. Phase Formation and Thermal Stability of CoCrFeNi and CoCrFeMnNi Equiatomic High Entropy Alloys. J. Alloys Compd. 2019, 774, p 856-864.

(32) He, F.; Wang, Z.; Wu, Q.; Li, J.; Wang, J.; Liu, C. T. Phase Separation of Metastable CoCrFeNi High Entropy Alloy at Intermediate Temperatures. Scr. Mater. 2017, 126, p $15-19$.

(33) Dahlborg, U.; Cornide, J.; Calvo-Dahlborg, M.; Hansen, T. C.; Fitch, A.; Leong, Z.; Chambreland, S.; Goodall, R. Structure of Some CoCrFeNi and CoCrFeNiPd Multicomponent HEA Alloys by Diffraction Techniques. J. Alloys Compd. 2016, 681, p 330-341.

(34) Sathiyamoorthi, P.; Basu, J.; Kashyap, S.; K G, P.; Kottada, R. S. Thermal Stability and Grain Boundary Strengthening in Ultrafine-Grained CoCrFeNi High Entropy Alloy Composite. Mater. Des. 2017, 134.

(35) Kini, M. K.; Lee, S.; Savan, A.; Breitbach, B.; Addab, Y.; Lu, W.; Ghidelli, M.; Ludwig, A.; Bozzolo, N.; Scheu, C.; Chatain, D.; Dehm, G. Nanocrystalline Equiatomic CoCrFeNi Alloy Thin Films: Are They Single Phase Fcc? Surf. Coat. Technol. 2021, 410, p 126945. 
(36) Munitz, A.; Salhov, S.; Hayun, S.; Frage, N. Heat Treatment Impacts the Micro-Structure and Mechanical Properties of AlCoCrFeNi High Entropy Alloy. J. Alloys Compd. 2016, 683, p 221-230.

(37) An, Z.; Jia, H.; Wu, Y.; Rack, P. D.; Patchen, A. D.; Liu, Y.; Ren, Y.; Li, N.; Liaw, P. K. Solid-Solution CrCoCuFeNi High-Entropy Alloy Thin Films Synthesized by Sputter Deposition. Mater. Res. Lett. 2015, 3 (4), p 203-209.

(38) Arfaoui, M.; Radnóczi, G.; Kovács Kis, V. Transformations in $\mathrm{CrFeCoNiCu} \mathrm{High} \mathrm{Entropy}$ Alloy Thin Films during In-Situ Annealing in TEM. Coatings 2020, 10 (1), p 60.

(39) Cui, P.; Ma, Y.; Zhang, L.; Zhang, M.; Fan, J.; Dong, W.; Yu, P.; Li, G.; Liu, R. Effect of Ti on Microstructures and Mechanical Properties of High Entropy Alloys Based on CoFeMnNi System. Mater. Sci. Eng. A 2018, 737, p 198-204.

(40) Shun, T.-T.; Chang, L.-Y.; Shiu, M.-H. Microstructures and Mechanical Properties of Multiprincipal Component CoCrFeNiTix Alloys. Mater. Sci. Eng. A 2012, 556, p 170174.

(41) Shun, T.-T.; Hsieh, C.-Y.; Hung, W.-J.; Lee, C.-F. Age Heat Treatment of the CoCrFeNiTi 0.3 High-Entropy Alloy. Mater. Trans. 2018, 59 (5), p 730-733.

(42) Pickering, E. J.; Muñoz-Moreno, R.; Stone, H. J.; Jones, N. G. Precipitation in the Equiatomic High-Entropy Alloy CrMnFeCoNi. Scr. Mater. 2016, 113, p 106-109.

(43) Reynolds, C. R.; Herl, Z.; Ley, N. A.; Choudhuri, D.; Lloyd, J. T.; Young, M. L. Comparing CALPHAD Predictions with High Energy Synchrotron Radiation X-Ray Diffraction Measurements during in Situ Annealing of A10.3CoCrFeNi High Entropy Alloy. Materialia 2020, 12, p 100784.

(44) Dolique, V.; Thomann, A.-L.; Brault, P.; Tessier, Y.; Gillon, P. Thermal Stability of AlCoCrCuFeNi High Entropy Alloy Thin Films Studied by In-Situ XRD Analysis. Surf. Coat. Technol. 2010, 204 (12-13), p 1989-1992.

(45) Gorsse, S.; Tancret, F. Current and Emerging Practices of CALPHAD toward the Development of High Entropy Alloys and Complex Concentrated Alloys. J. Mater. Res. 2018, 33 (19), p 2899-2923. 
Table 1. Structure and lattice parameter of the metallic element used in this work.

\begin{tabular}{|l|l|l|}
\hline Element & Structure & $\begin{array}{l}\text { Lattice parameter } \\
(\AA)\end{array}$ \\
\hline $\mathrm{Al}$ & $\mathrm{FCC}$ & 4.05 \\
\hline $\mathrm{Co}$ & $\mathrm{HCP}$ & $2.51 / 4.07$ \\
\hline $\mathrm{Cr}$ & $\mathrm{BCC}$ & 2.88 \\
\hline $\mathrm{Cu}$ & $\mathrm{FCC}$ & 3.61 \\
\hline $\mathrm{Fe}$ & $\mathrm{BCC}$ & 2.87 \\
\hline $\mathrm{Ni}$ & $\mathrm{FCC}$ & 3.52 \\
\hline $\mathrm{Ti}$ & $\mathrm{HCP}$ & $2.95 / 4.68$ \\
\hline
\end{tabular}

Table 2. Calculated lattice parameter $(\AA)$ of the CoFeNi and CoCrFeNi milled powders before and after annealing at different temperatures. The number in parentheses is the estimated standard deviation. For instance, 3.571(2) means $3.571 \pm 0.002$.

\begin{tabular}{|c|c|c|c|c|}
\hline \multirow{2}{*}{$\begin{array}{c}\text { System } \\
\text { (alloy) }\end{array}$} & $\begin{array}{c}\text { Initial, at room } \\
\text { temperature }\end{array}$ & \multicolumn{3}{|c|}{$\begin{array}{c}\text { Lattice parameter after annealing and cooling down to } \\
\text { room temperature }\end{array}$} \\
\cline { 3 - 5 } & & $873 \mathrm{~K}$ & $1073 \mathrm{~K}$ & $1273 \mathrm{~K}$ \\
\hline $\mathrm{CoFeNi}$ & $3.571(2)$ & $3.5711(2)$ & $3.5621(3)$ & $3.5620(3)$ \\
\hline $\mathrm{CoCrFeNi}$ & $3.575(4)$ & $3.5706(2)$ & $3.5748(1)$ & $3.5752(3)$ \\
\hline
\end{tabular}



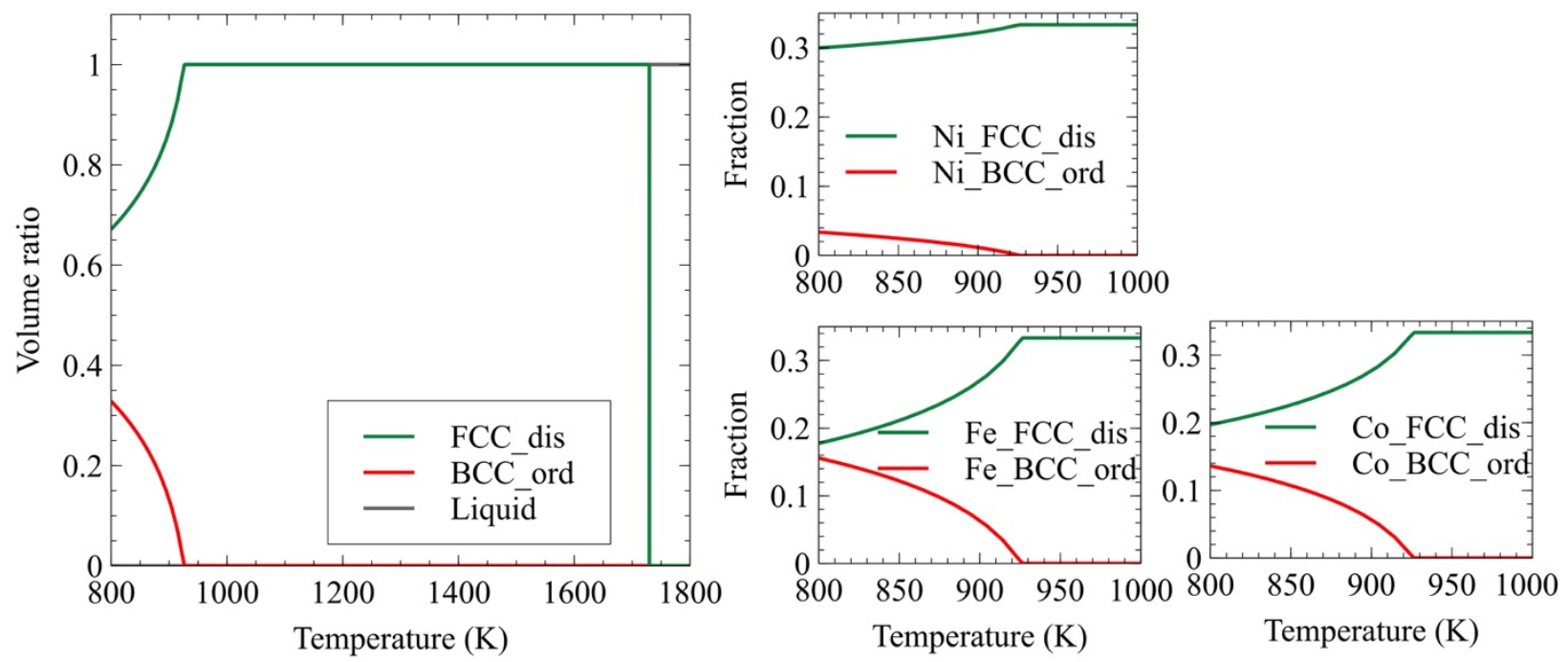

Figure 1. Phase fractions (volume fraction) in the CoFeNi system and element distributions in the different phases 

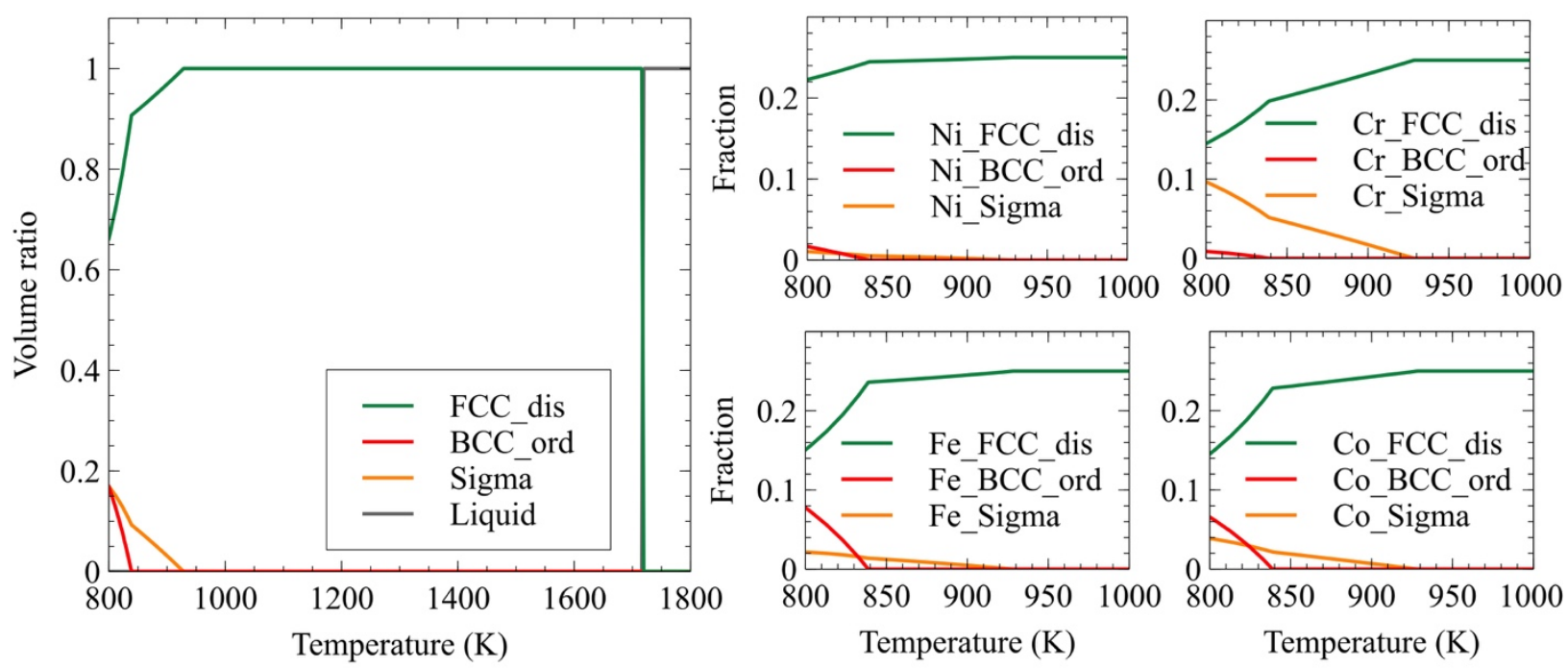

Figure 2. Phase fractions (volume percent) as a function of temperature in the equimolar $\mathrm{CoCrFeNi}$ system and element distributions in the different phases 
(a)

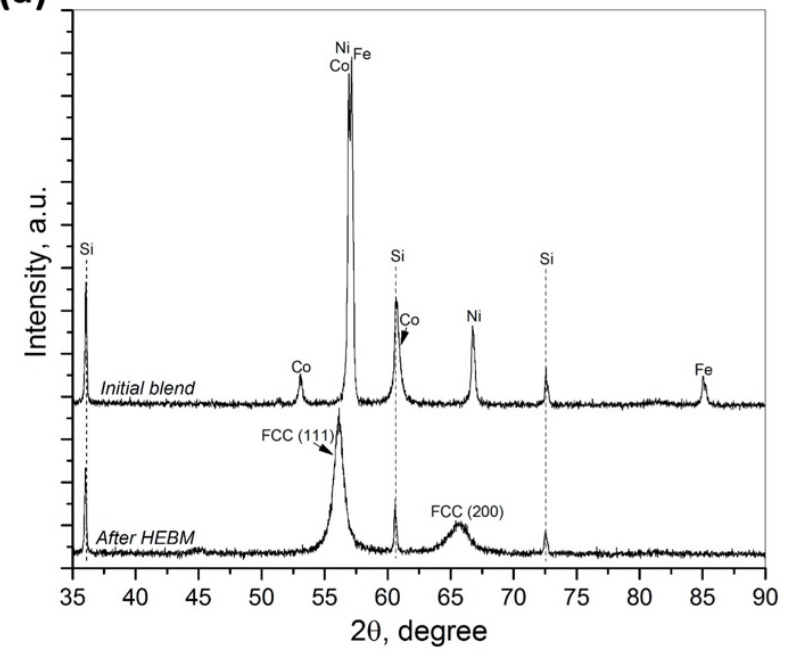

(b)

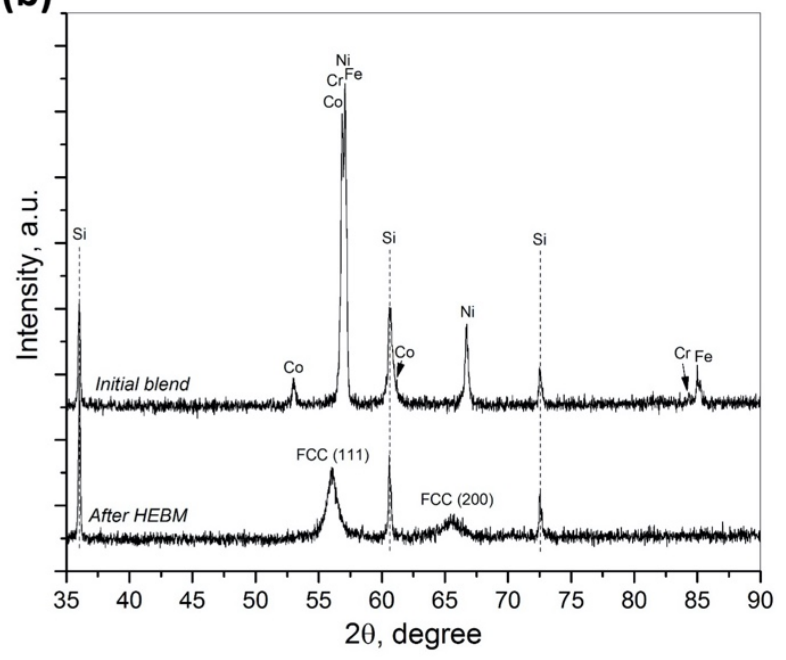

Figure 3. XRD patterns of the initial powder blend $\mathrm{Co}+\mathrm{Fe}+\mathrm{Ni}$ and mechanically alloyed $\mathrm{CoFeNi}$ (a) and $\mathrm{Co}+\mathrm{Cr}+\mathrm{Fe}+\mathrm{Ni}$ and mechanically alloyed $\mathrm{CoCrFeNi}$ (b) after 90 min of high energy ball milling ( $\mathrm{Fe}-\mathrm{K}_{\alpha}$ radiation). A powder of $\mathrm{Si}$ was added to the XRD samples in order to obtain reference peaks. 
(a)

(b)

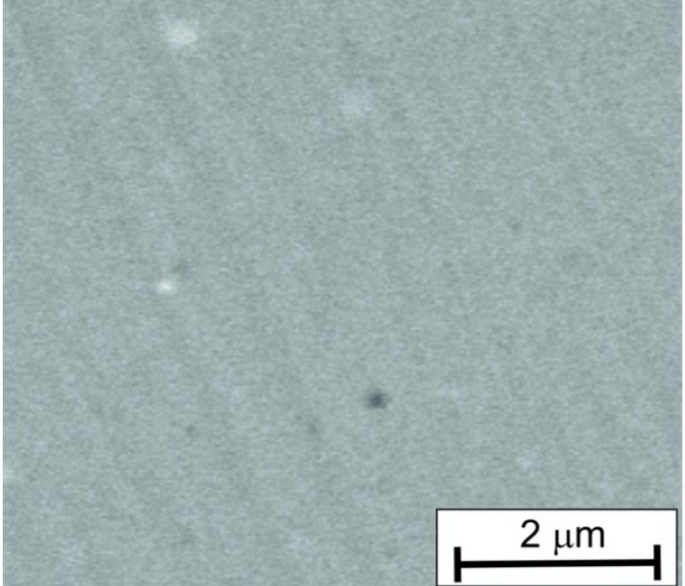

Figure 4. SEM images of the CoFeNi (a) and CoCrFeNi (b) alloys after 90 min of HEBM. Image in backscattered electrons. 
(a)

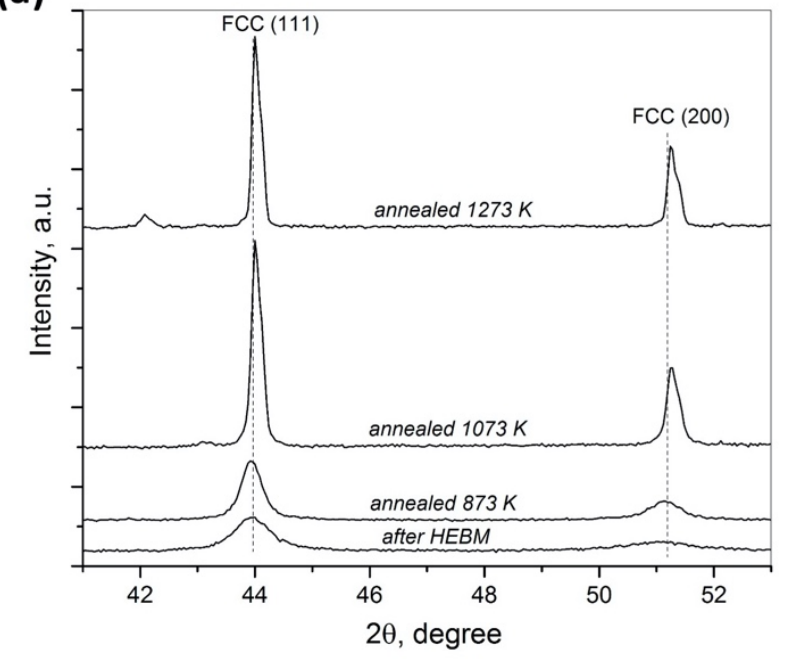

(b)

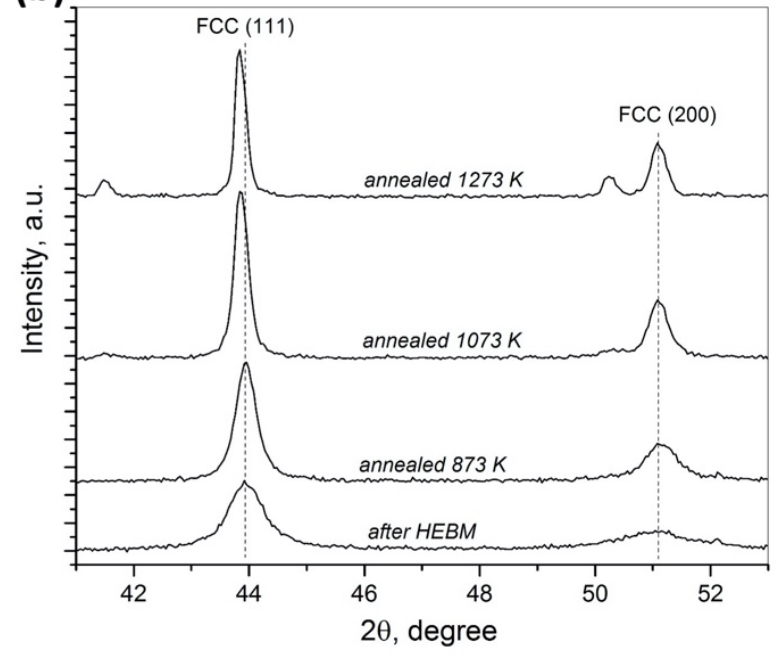

Figure 5. Room temperature XRD patterns of the $\mathrm{CoFeNi}$ (a) and $\mathrm{CoCrFeNi}$ (b) alloys after HEBM and after annealing at different temperatures over $5.1 \mathrm{~h}\left(\mathrm{Cu}-\mathrm{K}_{\alpha}\right.$ radiation $)$. 


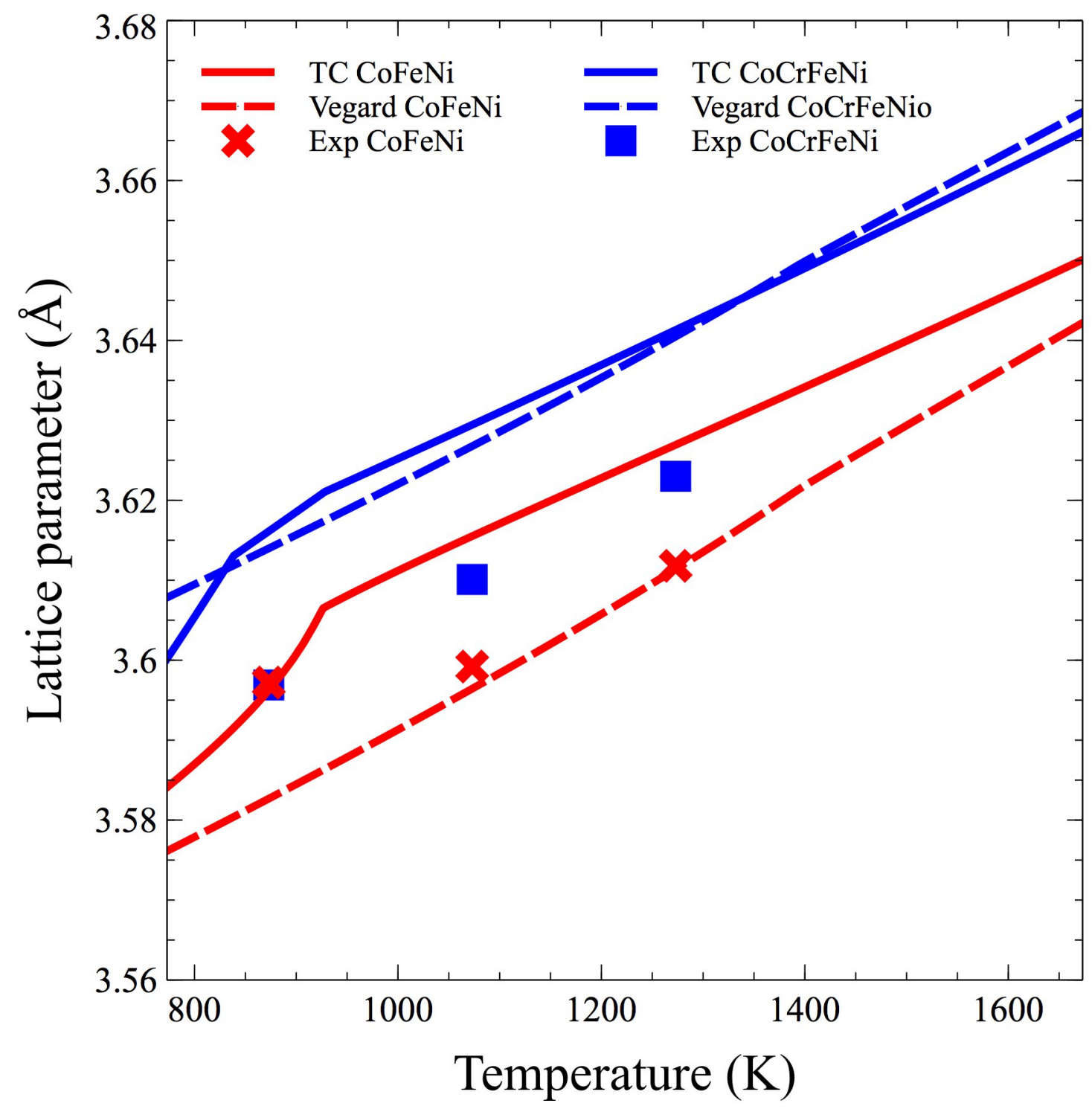

Figure 6. Lattice parameters for ternary and quaternary HEAs as a function of temperature. Only the FCC lattice has been selected. Experimental data were obtained by means of HT-XRD. 


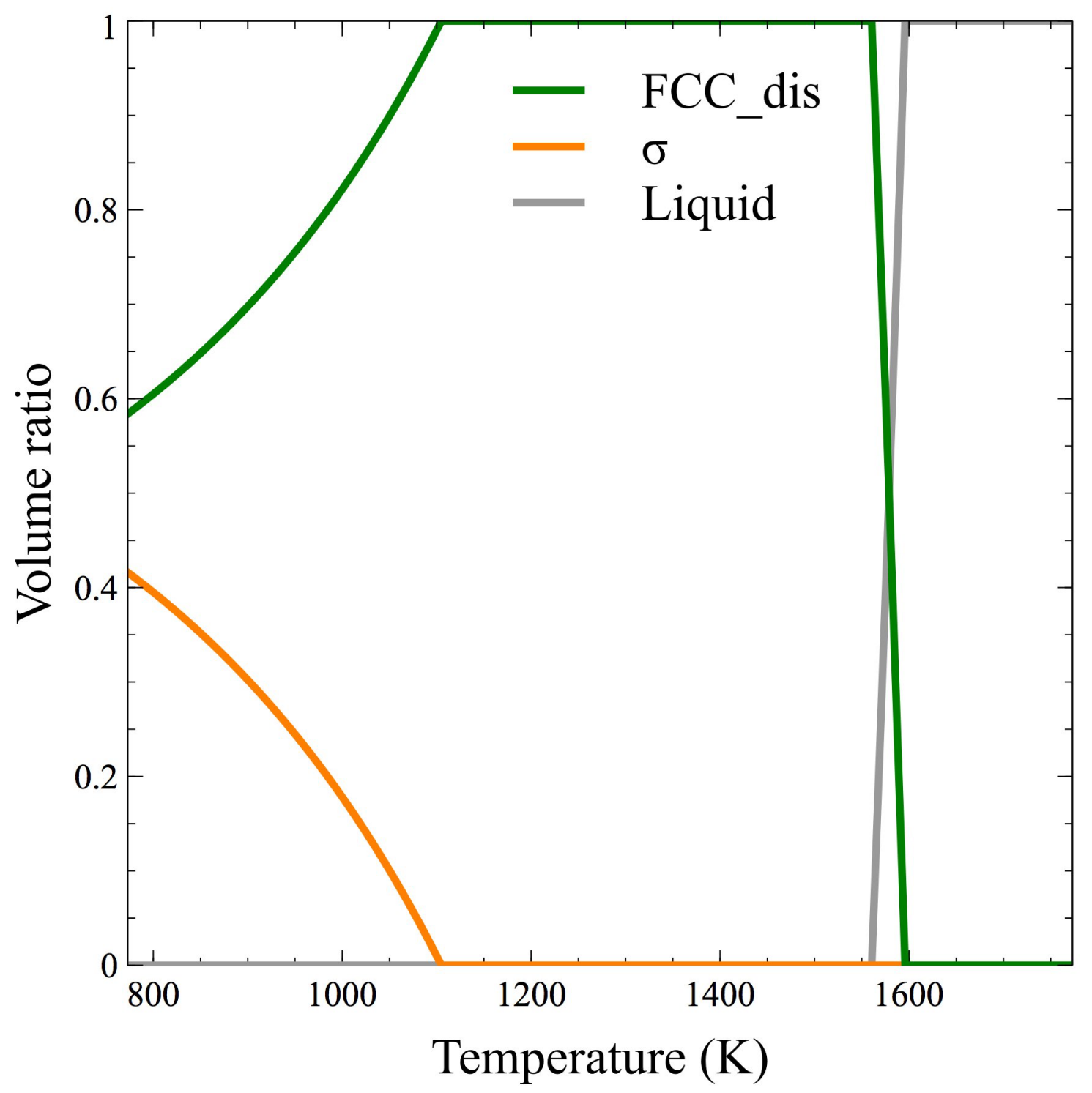

Figure 7. Volume ratios of the different phases in Mn-CoCrFeNi HEA calculated by Thermo-Calc. 


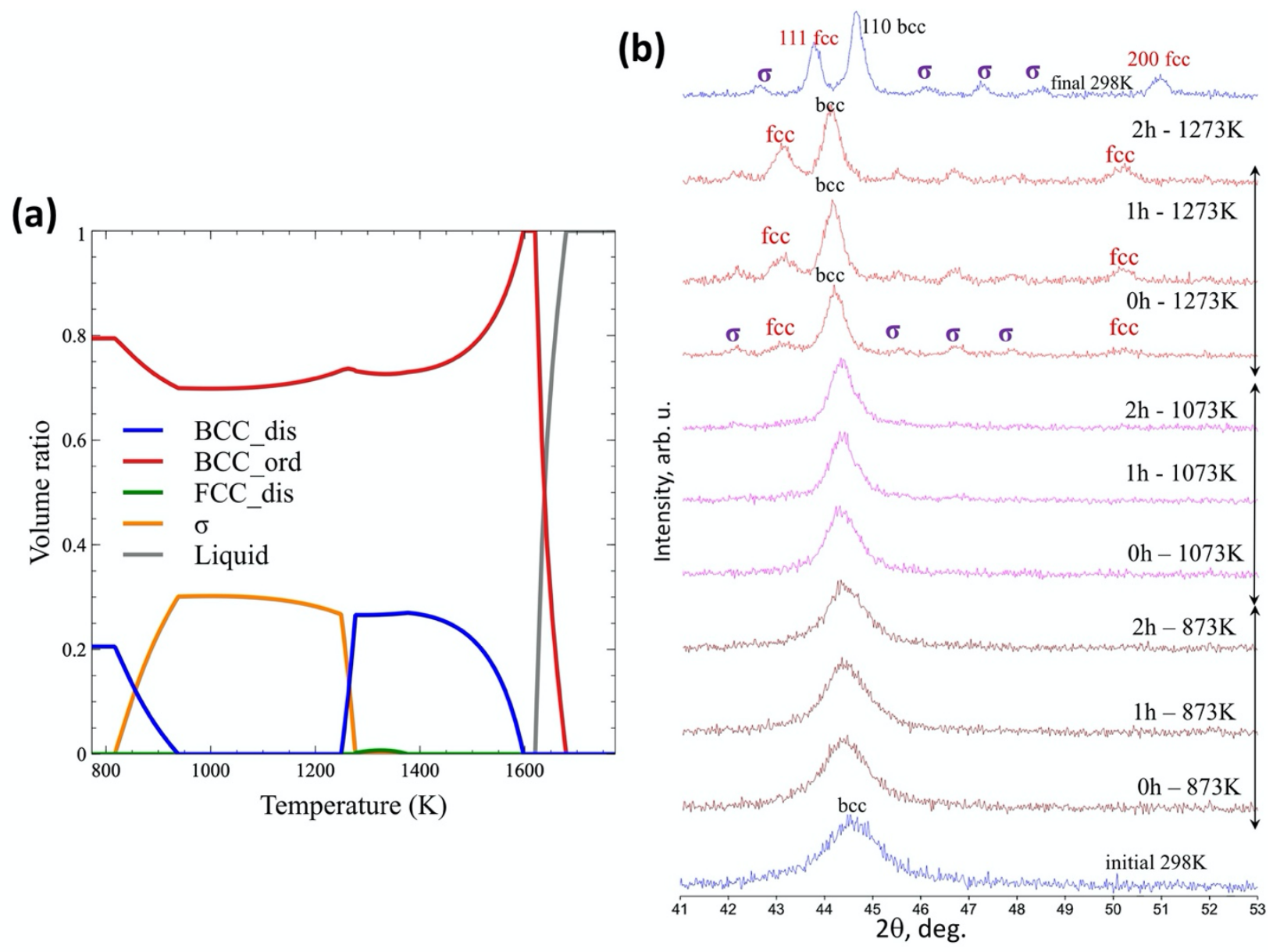

Figure 8. Volume ratios for the Al-CoCrFeNi alloy calculated using Thermo-Calc (a). High temperature XRD patterns after HEBM in Activator-2S (b). 


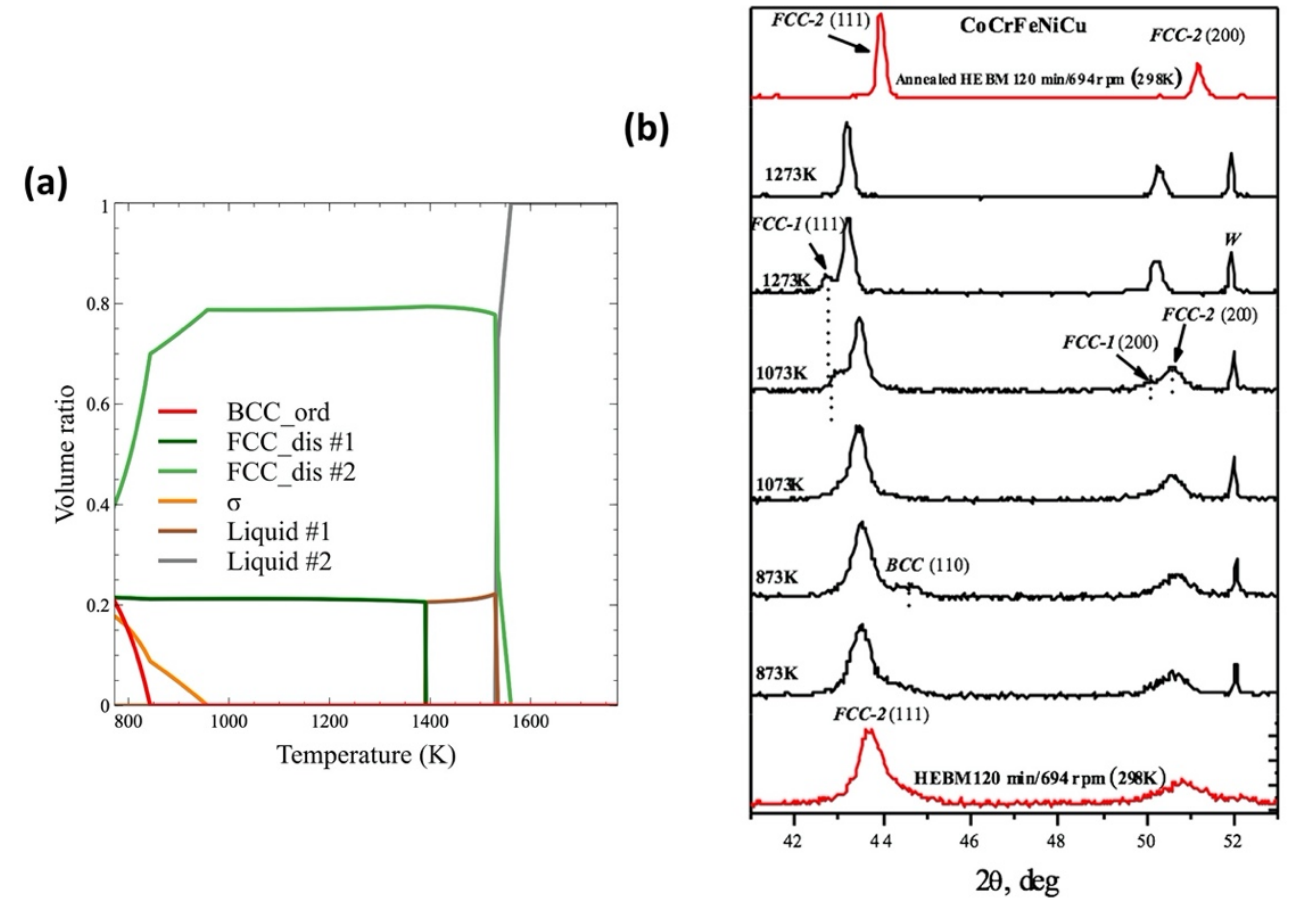

Figure 9. Volume ratios of phases for HEA Cu-CoCrFeNi calculated using Thermo-Calc (a). HTXRD of powder after HEBM (Activator-2S, 120 min of HEBM at 694/1388 rpm) (b). 


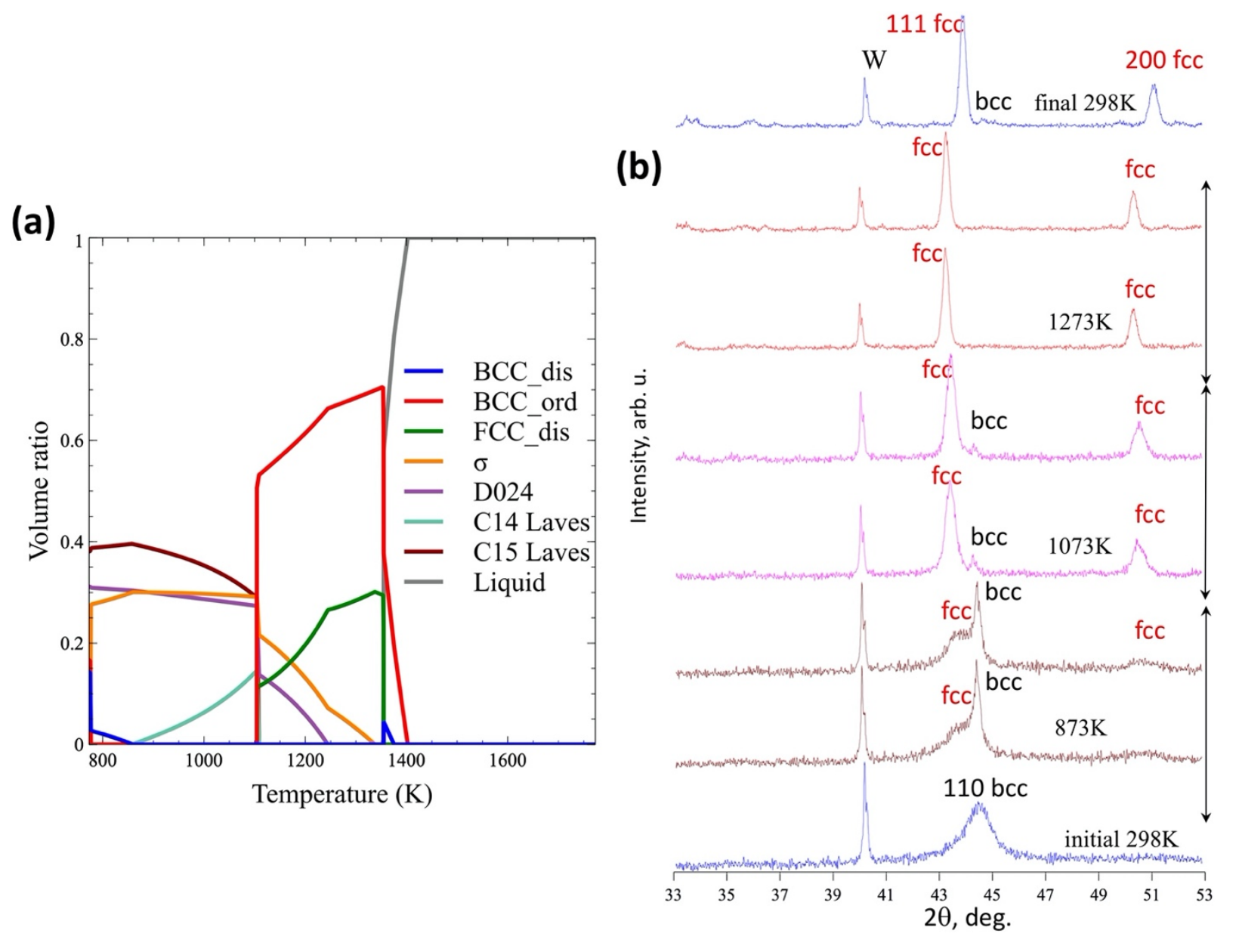

Figure 10. Volume ratios of phases for the Ti-CoCrFeNi alloy calculated using Thermo-Calc (a). High temperature XRD patterns of the powder after HEBM in AGO-2 (b). 


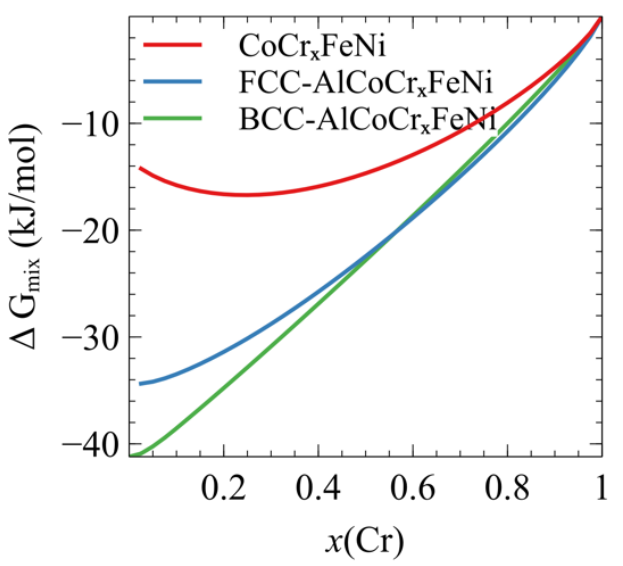

(a)

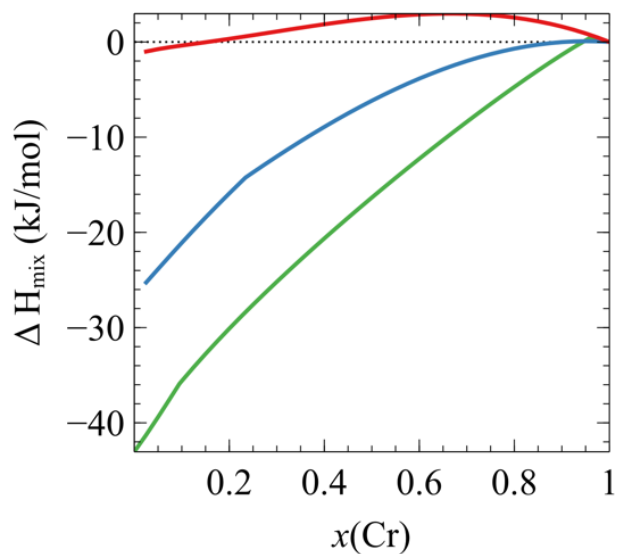

(b)

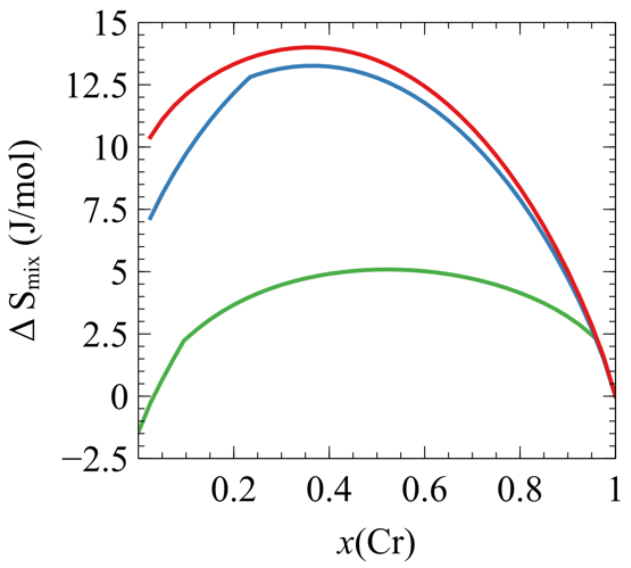

(c)

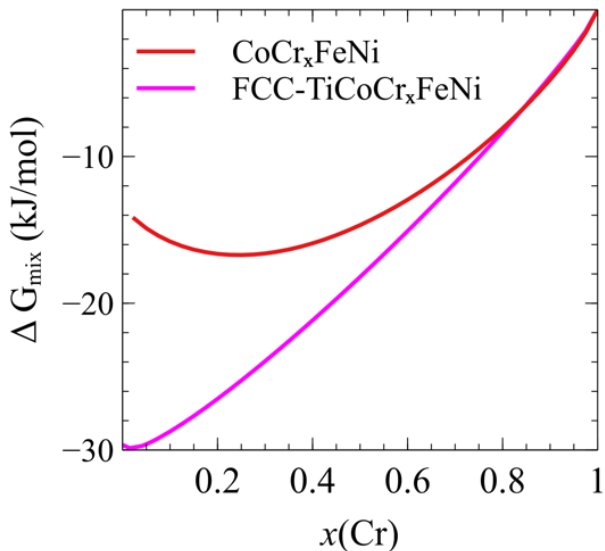

(d)

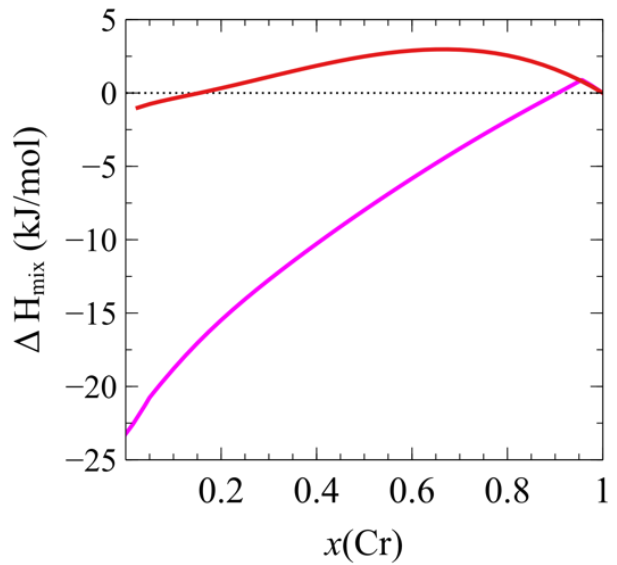

(e)

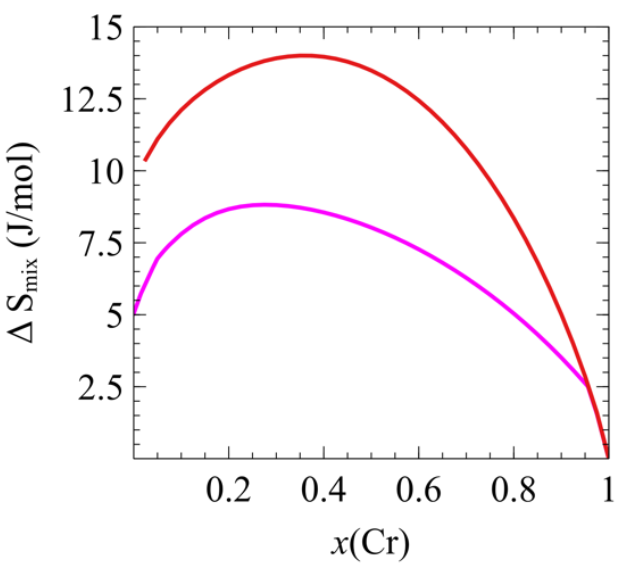

(f)

Figure 11. Calculated mixing properties of the $\mathrm{FCC}(\mathrm{BCC})$ phase at $1273 \mathrm{~K}$ : Gibbs free energy of mixing $\Delta G_{\mathrm{mix}}$, enthalpy of mixing $\Delta H_{\mathrm{mix}}$ and entropy of mixing $\Delta S_{\mathrm{mix}}$ as a function of Cr content for the quaternary $\mathrm{CoCr}_{\mathrm{x}} \mathrm{FeNi}$ and quinaries $\mathrm{Al}-\mathrm{CoCr}_{\mathrm{x}} \mathrm{FeNi}(\mathrm{a}-\mathrm{c})$ and $\mathrm{Ti}-\mathrm{CoCr}_{\mathrm{x}} \mathrm{FeNi}(\mathrm{d}-\mathrm{f})$. The reference state is $\mathrm{FCC}(\mathrm{BCC})$ at $\mathrm{T}=1273 \mathrm{~K}$. Thermo-Calc ${ }^{\circledR}$ with TCHEA3. 